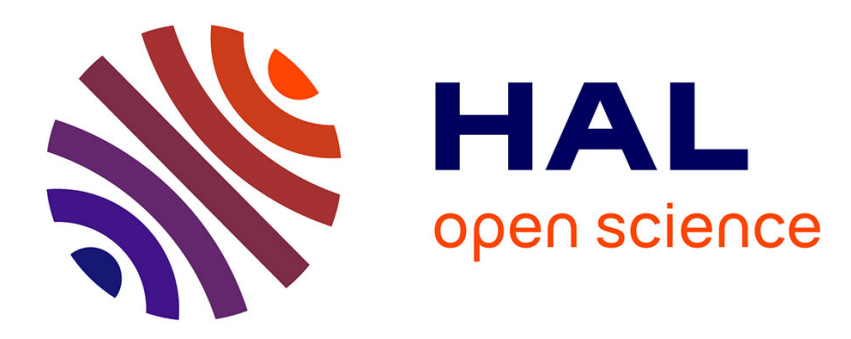

\title{
Moduli spaces for topologically quasi-homogeneous functions.
}

\author{
Yohann Genzmer, Emmanuel Paul
}

\section{To cite this version:}

Yohann Genzmer, Emmanuel Paul. Moduli spaces for topologically quasi-homogeneous functions.. Journal of Singularities, 2016. hal-00749661

\section{HAL Id: hal-00749661 \\ https://hal.science/hal-00749661}

Submitted on 8 Nov 2012

HAL is a multi-disciplinary open access archive for the deposit and dissemination of scientific research documents, whether they are published or not. The documents may come from teaching and research institutions in France or abroad, or from public or private research centers.
L'archive ouverte pluridisciplinaire HAL, est destinée au dépôt et à la diffusion de documents scientifiques de niveau recherche, publiés ou non, émanant des établissements d'enseignement et de recherche français ou étrangers, des laboratoires publics ou privés. 


\title{
Moduli spaces for topologically quasi-homogeneous functions.
}

\author{
Yohann Genzmer, Emmanuel PAuL
}

October 4, 2011

\begin{abstract}
We consider the topological class of a germ of 2-variables quasi-homogeneous complex analytic function. Each element $f$ in this class induces a germ of foliation $(f=$ constants) and a germ of curve $(f=0)$. We first describe the moduli space of the foliations in this class and give analytic normal forms. The classification of curves induces a distribution on this moduli space. By studying the infinitesimal generators of this distribution, we can compute the generic dimension of the moduli space for the curves, and we obtain the corresponding generic normal forms. 1
\end{abstract}

\section{Introduction}

From any convergent series $f$ in $\mathbb{C}\{x, y\}$, we can consider three different mathematical objects: a germ of holomorphic function defined by the sum of this series, a germ of foliation whose leaves are the connected components of the level curves $f=$ constants, and an imbedded curve $f=0$. Composing $f$ on the left side by a diffeomorphism of $(\mathbb{C}, 0)$ may change the function but nor the foliation or the curve. Multiplying $f$ by an invertible function $u$ may change the function and the foliation but not the related curve. Therefore, there are three different analytic equivalence relations:

- The classification of functions (or right equivalence):

$$
f_{0} \sim_{r} f_{1} \Leftrightarrow \exists \phi \in \operatorname{Diff}\left(\mathbb{C}^{2}, 0\right), f_{1}=f_{0} \circ \phi .
$$

- The classification of foliations (or left-right equivalence):

$$
f_{0} \sim f_{1} \Leftrightarrow \exists \phi \in \operatorname{Diff}\left(\mathbb{C}^{2}, 0\right), \psi \in \operatorname{Diff}(\mathbb{C}, 0), \psi \circ f_{1}=f_{0} \circ \phi .
$$

- The classification of curves:

$$
f_{0} \sim_{c} f_{1} \Leftrightarrow \exists \phi \in \operatorname{Diff}\left(\mathbb{C}^{2}, 0\right), \exists u \in \mathcal{O}_{2}, u(0) \neq 0, u f_{1}=f_{0} \circ \phi .
$$

In what follows, we are going to consider mostly the two last equivalence relations: as shown in [1], when $f$ has an isolated singularity, the difference between the two first equivalence relations are minor. Finally, we emphasize that in our work, we will always require that the conjugacies that appear above will respect a fixed numbering of the branches of $f=0$.

In the same way, one can define topological classifications requiring only topological changes of coordinates. The moduli space of a function, foliation or curve is the space of analytic classes in a given topological class.

\footnotetext{
${ }^{1}$ Keywords: holomorphic foliation, moduli of curve, singularities.

A.M.S. class.: $34 \mathrm{M} 35,32 \mathrm{~S} 65,32 \mathrm{G} 13$
} 
In what follows, we will denote by $\mathcal{T}$ the topological class of a quasi-homogeneous function: we recall that a germ of holomorphic function $f:\left(\mathbb{C}^{2}, 0\right) \rightarrow(\mathbb{C}, 0)$ is quasihomogeneous if and only if $f$ belongs to the jacobian ideal $J(f)=\left(\frac{\partial f}{\partial x}, \frac{\partial f}{\partial y}\right)$. If $f$ is quasi-homogeneous, there exist coordinates $(x, y)$ and positive coprime integers $k$ and $l$ such that the quasi-radial vector field $R=k x \frac{\partial}{\partial x}+l y \frac{\partial}{\partial y}$ satisfies $R(f)=d \cdot f$, where the integer $d$ is the quasi-homogeneous degree of $f$ [14]. In these coordinates, $f$ has $p$ cuspidal branches and maybe axial branches, that is to say, $f$ is written

$$
f=x^{n_{0}} y^{n_{\infty}} \prod_{b=1}^{p}\left(y^{k}+a_{b} x^{l}\right)^{n_{b}}
$$

where the multiplicities satisfy $n_{0} \geq 0, n_{\infty} \geq 0$ and $n_{b}>0$. The complex numbers $a_{b}$ are non vanishing numbers such that $a_{b} \neq a_{b^{\prime}}$. Using a convenient analytic change of coordinates, we may suppose that $a_{1}=1$.

A germ of holomorphic function $f$ is topologically quasi-homogeneous if the function $f$ is topologically conjugated to a quasi-homogeneous function. Therefore, following [11] and [3] its desingularization by blowing up's is the same as a quasi-homogeneous function, that is to say: the exceptional divisor is a chain of components isomorphic to $P^{1}(\mathbb{C})$, the strict transform of the cuspidal branches intersect the same component, the principal component, and the strict transform of the axes, if they appear, intersect the end components of this chain (see Appendix A and figure (6)). The previous expression (1) is a topological normal form for such a function.

A topologically quasi-homogeneous function is not necessarly quasi-homogeneous. Actually, if we fix the analytic invariants $a_{b}$ which corresponds to cross-ratios between the cuspidal branches on the principal component, the analytic class of a quasi-homogeneous function is unique in its topological class $\mathcal{T}[14]$.

The first aim of this paper is to describe the moduli space of a foliation induced by a topologically quasi-homogeneous function which is defined by the quotient

$$
\mathcal{M}=\mathcal{T} / \sim
$$

We give the infinitesimal description of this moduli space by making use of the cohomological tools considered by J.F. Mattei for any germ of foliation in [12]: the tangent space to the moduli space is given by the first Cech cohomology group $H^{1}\left(D, \Theta_{\mathcal{F}}\right)$, where $D$ is the exceptional divisor of the desingularization of $f$, and $\Theta_{\mathcal{F}}$ is the sheaf of germs of vector fields tangent to the desingularized foliation. Using a particular covering of $D$, we give a triangular presentation of the $\mathbb{C}$-space $H^{1}\left(D, \Theta_{\mathcal{F}}\right)$ in Theorem (1.1). This description leads us to consider triangular normal forms

$$
N_{a}=x^{n_{0}} y^{n_{\infty}} \prod_{b=1}^{p}\left(y^{k}+\sum_{\Phi(b, d) \in \mathbb{T}} a_{b, d} m^{d}\right)^{n_{b}}
$$

by perturbing the topological normal (1) with some monomials following an algorithm described in the subsection (1.2). This family of normal forms turns out to be semiuniversal as established in Theorem (1.10). In this way, we obtain a local description of $\mathcal{M}$ By a compacity argument, we finally give a global description of this moduli space in Theorem (1.15) and Theorem (1.16) by proving that any function in $\mathcal{T}$ is actually conjugated to some normal form $N_{a}$, and that the parameter $a$ is unique up to some weighted projective action of $\mathbb{C}^{*}$.

All the results of this first part can be extended to the generic Darboux function:

$$
f^{(\lambda)}=f_{1}^{\lambda_{1}} \cdots f_{p}^{\lambda_{p}}
$$


with complex multiplicities $\lambda_{i}$. Nevertheless, we do not insert this extension here, since we have previously explain in [8] how to perform it in the topologically homogeneous case.

The second part of our work is dedicated to the study of the moduli space of curves in the quasi-homogeneous topological class. This problem is a particular case of an open problem known as the Zariski problem. It has only a very few satisfying answer: Zariski [16] for the very first treatment of some particular cases, Hefez and Hernandez [5, 6] for the irreducible curves, Granger [9] in the homogeneous topological class and [2] for some results which are particular case of our present results. Our strategy that we already introduced in a previous work [8], differs from all this works: from our description of the moduli space $\mathcal{M}$, we consider the distribution $\mathcal{C}$ on $\mathcal{M}$ induced by the equivalence relation $\sim_{c}$ : two foliations represented by two points in $\mathcal{M}$ are in a same orbit of this distribution if and only if they induce the same curve up to analytic conjugacy. Studying the familly of vector fields that induce the distribution $\mathcal{C}$ on $\mathcal{M}$, we compute the dimension of the generic strata of the moduli space of curves $\mathcal{M} / \mathcal{C}$ in Theorem (2.13). We give also an algorithm in order to construct the corresponding generic normal forms in Theorem (2.14).

In order to keep a sufficiently readable text, we have postponed a lot of technical computations in appendix.

\section{Contents}

1 The moduli space of foliations.

1.1 The infinitesimal description. . . . . . . . . . . . . . . . . 3

1.2 Construction of the local normal forms. . . . . . . . . . . . . . 6

1.3 Local universality . . . . . . . . . . . . . . . . . . . . . . . . . . . . . . . . . . . . . . . . . . .

1.4 The global moduli space of foliations. . . . . . . . . . . . . 15

2 The moduli space of curves. 19

2.1 The infinitesimal generators of $\mathcal{C} \ldots \ldots \ldots$. . . . . . . . . . . . . . . . . . . . . . . . .

2.2 The dimension of the generic strata. . . . . . . . . . . . . . . . . . . . . . . . . . . . . . 20

2.3 Normal forms for curves. . . . . . . . . . . . . . . . . . . . . . . 24

2.4 An example: the case $y^{n}+x^{n+1} \ldots \ldots \ldots \ldots$

3 Appendix $\quad 26$

3.1 A: reduction of singularities of a quasi-homogeneous function . . . . . . . 26

3.1.1 The algorithm of desingularization . . . . . . . . . . . . . . 27

3.1.2 Atlas of charts induced by the algorithm. . . . . . . . . . . . 27

3.1.3 Properties of the principal component. . . . . . . . . . . . . 28

3.1.4 Computing multiplicities . . . . . . . . . . . . . . . . 29

3.1.5 Properties of the sheaf $\Theta_{\mathcal{F}} \ldots \ldots \ldots \ldots$. . . . . . . . . 31

3.2 B: complement on the study of the vector fields $X_{m, n} \ldots \ldots \ldots 32$

3.3 C: normal forms for $(k, l)=(3,5)$ and $p=4 \ldots \ldots . \ldots . \ldots$

\section{The moduli space of foliations.}

\subsection{The infinitesimal description.}

We fix any class of function $f$ in $\mathcal{T}$ which is not necessarily the quasi-homogeneous topological model. Let $\mathcal{F}$ be the foliation defined by $f \circ E$ where $E: M \rightarrow\left(\mathbb{C}^{2}, 0\right)$ is the composition of the blowing up's appearing in its desingularization process. Let $D=E^{-1}(0)$ 
be the exceptional divisor.

Since the transverse structure of a foliation defined by a function is rigid, i.e. completely given by the discrete data of the multiplicities, any topological deformation is an unfolding as defined in [12]. We know from the same reference that the tangent space to the moduli space of unfoldings of a foliation $\mathcal{F}$ is the vector space: $H^{1}\left(D, \Theta_{\mathcal{F}}\right)$, where $\Theta_{\mathcal{F}}$ is the sheaf on $D$ of germs of holomorphic vector fields tangent to the desingularized foliation. Furthermore, this vector space in a finite dimensional one, and gave a formula for its dimension $\delta$ involving the multiplicities of the foliation at the singular points appearing at each step of the blowing up process. In the present topological class, we will give a alternative description of this tangent space which will allow us to construct normal forms.

Theorem 1.1. There is a one to one correspondance between $H^{1}\left(D, \Theta_{\mathcal{F}}\right)$ and the $\mathbb{C}$ vector space generated by the integer points $e_{i, j}$ in the triangle $\mathbb{T}$ in the upperhalf plane $(i, j), j \geq 0$, strictly delimited by the two lines of equations

$$
\begin{aligned}
k i-(k-v)\left(j-\nu_{c}\right) & =0 \\
l i-(l-u)\left(j-\nu_{c}\right) & =0
\end{aligned}
$$

where $\nu_{c}=k l p-k-l+l \varepsilon_{0}+k \varepsilon_{\infty}$ is the multiplicity of the foliation on the principal component of $D$ - see Proposition (3.1) in Appendix A for the value of $\varepsilon_{0}$ and $\varepsilon_{\infty}-$, and $u$ and $v$ are defined by the Bézout identity: $u k-v l=1,0 \leq u<l, 0 \leq v<k$.

We give a presentation of the tangent space to the moduli space of a function in the topological class: $(k, l)=(3,5), p=4, n_{0}=n_{\infty}=0, n_{1}=\cdots=n_{4}$ arbitrary, in Figure (6).

Proof. We will make use of the notations of Appendix A: $D_{1}, \ldots D_{c}, \ldots D_{N}$ is the chain of components of the exceptional divisor, $D_{c}$ the principal component, $\theta_{f}$ the vector field with isolated singularities

$$
\left(\frac{-\partial f}{\partial y}, \frac{\partial f}{\partial x}\right) / g . c . d .\left(\frac{-\partial f}{\partial y}, \frac{\partial f}{\partial x}\right) .
$$

Let $U_{0}$ be an open set in $M$ which covers $\cup_{i=1}^{c}\left(D_{i}\right)$ excepted a small disc around $D_{c} \cap D_{c+1}$ in $D_{c}$, and $U_{\infty}$ an open set covering $\cup_{i=c}^{N}\left(D_{i}\right)$ excepted a small disc around $D_{c-1} \cap D_{c}$ in $D_{c}$. Let $\mathcal{U}$ be the covering of $D$ by these two open sets $U_{0}$ and $U_{\infty}$ - see figure (5) in Appendix A. We know from the proposition (3.6) of the Appendix A that $H^{1}\left(D, \Theta_{\mathcal{F}}\right)=H^{1}\left(\mathcal{U}, \Theta_{\mathcal{F}}\right)$. Therefore we have

$$
H^{1}\left(D, \Theta_{\mathcal{F}}\right)=\frac{\Theta_{\mathcal{F}}\left(U_{0} \cap U_{\infty}\right)}{\Theta_{\mathcal{F}}\left(U_{0}\right) \oplus \Theta_{\mathcal{F}}\left(U_{\infty}\right)}
$$

In order to compute each term of this quotient, we consider the chart $\left(x_{c}, y_{c}\right)$ of $D_{c}$ (see the atlas of charts defined in 3.1.2). The vector field

$$
\theta_{i s}=\frac{E^{*} \theta_{f}}{y_{c}^{\nu_{c}}}
$$

has isolated singularities, and defines $\mathcal{F}$ on $U_{0} \cap U_{\infty}$. Therefore we have:

$$
\Theta_{\mathcal{F}}\left(U_{0} \cap U_{\infty}\right)=\left\{\sum_{i \in \mathbb{Z}, j \in \mathbb{Z}^{+}} \lambda_{i, j} x_{c}^{i} y_{c}^{j} \cdot \theta_{i s}\right\}
$$

By the local monomial expression of $E$ given by Proposition (3.1) in Appendix A, these vector fields $\theta$ blow down on meromorphic vector fields with poles on the axes:

$$
E_{*} \theta=\sum_{i \in \mathbb{Z}, j \in \mathbb{Z}^{+}} \lambda_{i, j} x^{i l-j(l-u)+\nu_{c}(l-u)} y^{-i k+j(k-v)-\nu_{c}(k-v)} \cdot \theta_{f} .
$$


Lemma 1.2. A vector field $\theta$ in $\Theta_{\mathcal{F}}\left(U_{0} \cap U_{\infty}\right)$ extends to $\Theta_{\mathcal{F}}\left(U_{0}\right)$-resp. to $\Theta_{\mathcal{F}}\left(U_{\infty}\right)-$ if and only if the meromorphic vector field $E_{*} \theta$ has an holomorphic extension on the $y$-axis -resp. $x$-axis-.

Proof. Clearly, if $\theta$ has an holomorphic extension to $\Theta_{\mathcal{F}}\left(U_{0}\right)$ which contains the strict transform of the $y$-axis, its blow-down has an holomorphic extension on the $y$-axis. On the converse, if $E_{*} \theta$ has such extension on the $y$-axis, $\theta$ is an holomorphic vector field on $U_{0} \cap U_{\infty}$ whose meromorphic extension on $U_{0}$ is holomorphic around the end point $\left(D_{1}, 0\right)$. We claim that this vector field is holomorphic on the whole open set $U_{0}$. Indeed, if the multiplicities $\alpha_{1}$ and $\alpha_{c}$ of this vector field on $D_{1}$ and $D_{c}$ are positive, the intermediate multiplicities $\alpha_{i}, 1<i<c$ are also positive. This is a consequence of the relations

$$
\alpha_{2}=e_{1} \alpha_{1}, \alpha_{i+1}=e_{i} \alpha_{i}-\alpha_{i-1}, i=2, \cdots c-1
$$

which can be obtained by using the change of charts, or by a similar argument as in proposition (3.3). Since $e_{i} \geq 2$ for $i=1, \ldots c-1$, we have

$$
\alpha_{2} \geq \alpha_{1}, \alpha_{i+1}-\alpha_{i}=\left(e_{i}-1\right) \alpha_{i}-\alpha_{i-1} \geq \alpha_{i}-\alpha_{i-1}, \quad i=2, \cdots c-1
$$

which proves that this sequence increases.

End of the proof of Theorem (1.1). From the previous lemma we deduce that an element $\theta$ in $\Theta_{\mathcal{F}}\left(U_{0} \cap U_{\infty}\right)$ belongs to $\Theta_{\mathcal{F}}\left(U_{0}\right)$ if and only if $-i k+j(k-v)-\nu_{c}(k-v) \geq 0$ and it belongs to $\Theta_{\mathcal{F}}\left(U_{\infty}\right)$ if and only if $i l-j(l-u)+\nu_{c}(l-u) \geq 0$. Therefore, there is an explicit bijection $\Psi$ between the $\mathbb{C}$-space $\mathbb{T}_{\mathbb{C}}$ generated by the integer points $e_{i, j}$ in $\mathbb{T}$ and $H^{1}\left(D, \Theta_{\mathcal{F}}\right)$ defined by:

$$
\Psi: \sum_{(i, j) \in \mathbb{T}} \lambda_{i, j} e_{i, j} \longmapsto\left[\sum_{(i, j) \in \mathbb{T}} \lambda_{i, j} x_{c}^{i} y_{c}^{j} \cdot \theta_{i s}\right]
$$

Remark 1.3. The two vectors $(k-v, k)$ and $(l-u, l)$ are tangent to the lines which bound the triangle $\mathbb{T}$. One can easily check that $H^{1}\left(D, \Theta_{\mathcal{F}}\right)$ has a structure of $\mathcal{O}_{2}$-module. Since we have $x=x_{c}^{k-v} y_{c}^{k}$ and $y=x_{c}^{l-u} y_{c}^{l}$, the multiplication by $x$ or $y$ acts on this representation by translations of these vectors. That is why we adopt the notation

$$
\vec{x}=(k-v, k) \text { and } \vec{y}=(l-u, l) .
$$

The two lines cut the vertical axis $(i=0)$ at the point $\left(0, \nu_{c}\right)$. The triangular representation of $H^{1}\left(D, \Theta_{\mathcal{F}}\right)$ is completely determined by this point $\left(0, \nu_{c}\right)$ and the two directions $\vec{x}$ and $\vec{y}$ (see Figure (6)).

This representation gives us a direct formula for the dimension $\delta$ of $H^{1}\left(D, \Theta_{\mathcal{F}}\right)$, by counting the integers points in the above triangle. In order to give an explicit formula, we need the following fact (whose proof is left to the reader):

Lemma 1.4 (and notations). The number of integer points in an open interval $] a, b[$ is given by $] b]-[a[$, where $[a[$ stands for the usual integer part $n$ of $a: n \leq a<n+1$, and $] b]$ is the "strict" integer part $m$ of $b$ defined by $m<b \leq m+1$.

Since the intersections of the horizontal levels $j$ with the two lines are given by $a_{j}=$ $\frac{k-v}{k}\left(j-\nu_{c}\right)$ and $b_{j}=\frac{l-u}{l}\left(j-\nu_{c}\right)$ we obtain 
Proposition 1.5. Let $\nu_{c}=k l p-k-l+k \varepsilon_{0}+l \varepsilon_{\infty}$ be the multiplicity of $\mathcal{F}$ on the principal component of $D$. The dimension of $H^{1}\left(D, \Theta_{\mathcal{F}}\right)$ is

$$
\left.\left.\delta=\sum_{j=0}^{\nu_{c}}\right] \frac{l-u}{l}\left(j-\nu_{c}\right)\right]-\left[\frac{k-v}{k}\left(j-\nu_{c}\right)[.\right.
$$

Example. For the topological class given by $(k, l)=(3,5), p=4$, without axis, by counting the integers points in figure (6) in Appendix C, or applying the previous formula, we obtain that $\delta=78$.

\subsection{Construction of the local normal forms.}

We will construct here analytic models for topologically quasi-homogeneous functions starting from the topological normal form (1). Since it already appears $(p-1)$ analytic invariants that is the value $a_{b}$, we have to add $\delta-(p-1)$ monomial terms of higher degree. The construction to come is a priori based upon some algorithmic but arbitrary choices. It will be justified by Theorem (1.10) in the next section.

In our previous work in [8], for the homogeneous topological class, in which the topological representative was $p$ transverse lines, we straightened the fourth first lines on $x y(y+x)\left(y+a_{4,1} x\right)$, added the monomials $a_{5,2} x^{2}$ to the fifth line, $a_{6,2} x^{2}+a_{6,3} x^{3}$ to the sixth, and so on. We generalize this triangular construction here by making use of the quasi-homogeneous $(k, l)$-degree. Nevertheless, the choice of the monomials and their distribution between the branches is not so obvious here.

The figure (6) in Appendix $\mathrm{C}$ shows the procedure in order to construct the normal forms associated to the topological class of

$$
\left(y^{3}+x^{5}\right)^{n_{1}}\left(y^{3}+a_{2} x^{5}\right)^{n_{2}}\left(y^{3}+a_{3} x^{5}\right)^{n_{3}}\left(y^{3}+a_{4} x^{5}\right)^{n_{4}} .
$$

The meaning of all the datas that appear on the figure will be detailed below.

The construction consists in three successive steps.

Step 1. The triangle of moduli. First, one has to draw the triangle $\mathbb{T}$ in the half plane $(i, j), j \geq 0$, bounded by the two lines given by Theorem (1.1).

Example. For the topological type $(3,5)$ with multiplicities $\left(0,0, n_{1}, n_{2}, n_{3}, n_{4}\right)$ considered in Figure (6), since $2 \times 3-1 \times 5=1$, we obtain that $u=2$ and $v=1$. We have $\nu_{c}=15 \times 4-5-3=52$. Therefore the triangle is bounded by the lines:

$$
5 i-3(j-52)=0, \quad 3 i-2(j-52)=0 .
$$

Step 2. Choice of the monomials. Let $d_{0}=k l$ the quasi-homogeneous degree of each cuspidal branch $y^{k}-a_{b} x^{l}$. One can easily check the following fact :

Lemma 1.6. For any $d \geq d_{0}$, there exists a unique monomial $x^{i} y^{j}$ with quasi-homogeneous degree $d$, such that $j<k$. We denote it: $m^{d}$.

Example. For $(k, l)=(3,5)$, we find $m^{15}=x^{5}, m^{16}=x^{2} y^{2}, m^{17}=x^{4} y, m^{18}=x^{6}, \ldots$

Therefore, to each horizontal line of index $j$, one can associate the monomial $m^{d}, d=k l+j$. We put them on a column on the right side in Figure (6).

Step 3. Choice of the branches. Now we will distribute these monomials between the $p$ cuspidal branches. The link between the monomial terms $m^{d}$ and $m^{d+1}$ is the multiplication by the meromorphic monomial term $x^{i} y^{j}=m^{d+1} / m^{d}$. According to remark (1.3), this multiplication induces the translation $\overrightarrow{t_{d}}=i \vec{x}+j \vec{y}$ between two consecutive lines of the upper half plane. 
Lemma 1.7. For any $d$, the components of the translations $\overrightarrow{t_{d}}$ in the canonical basis of the upper half plane $(i, j)$ are either $(1,1)$ or $(0,1)$.

Proof. Let $m^{d}=x^{i} y^{j}$ and thus $i k+j l=d$ with $0 \leq j<k$. Suppose first that $j-v \geq 0$. Then $m^{d+1}=x^{i+u} y^{j-v}$. Hence, in the the canonical basis, the components of $\overrightarrow{t_{d}}$ are

$$
(i+u-i)(k-v, k)+(j-v-j)(l-u, l)=(1,1) .
$$

If $j-v<0$ then $m^{d+1}=x^{i+u-l} y^{j+k-v}$. Indeed, we have $0 \leq j+k-v<k$ and $i+u-l \geq 0$ since from

$$
(i+u) k=k l+1-(j-v) l>k l .
$$

In this case, the components of $\overrightarrow{t_{d}}$ are

$$
(u-l)(k-v, k)+(k-v)(l-u, l)=(0,1)
$$

Example. The meromorphic monomials form a periodic sequence of lenght 3 generated by: $y^{2} / x^{3}, x^{2} / y, x^{2} / y$. The successive translations are $\overrightarrow{t_{15}}=2 \vec{y}-3 \vec{x}, \overrightarrow{t_{16}}=2 \vec{x}-\vec{y}$, $\overrightarrow{t_{17}}=2 \vec{x}-\vec{y}, \overrightarrow{t_{18}}=\overrightarrow{t_{15}}$ etc..., whose components are $(0,1),(1,1),(1,1)$. We put the translations on a column on the right side of Figure (6).

Now we consider all the parallel paths issued from the integer points $(i, 0)$ on the horizontal axe, under the action of the successive translations $\overrightarrow{t_{d}}$. Let $(c, 0)$ be the intersection of the left edge of the triangle with this horizontal axe. We consider the $p$ integer points:

$$
M_{1}:=\left(\left[c[+p, 0), M_{2}:=\left(\left[c[+p-1,0), \ldots M_{p}:=([c[+1,0) .\right.\right.\right.\right.
$$

Notice that the $(p-1)$ last ones are inside the triangle, while the first one is outside.

Proposition 1.8. The $p$ paths issued from the initial points $M_{i}, i=1, \cdots p$, obtained by the action of the successive translations $\overrightarrow{t_{d}}$ pass through all the integer points inside the triangle $\mathbb{T}$.

Proof. Let $i_{n}$ and $j_{n}$ such that $m^{d_{0}+n}=x^{i_{n}} y^{j_{n}}$. Following the arguments in the proof of Lemma (1.7), the sequence $\left(i_{n}, j_{n}\right)$ is explicitely defined by the following system

$$
\begin{cases}i_{n} & =l+u a_{n}-(l-u) b_{n} \\ j_{n} & =-v a_{n}+(k-v) a_{n} \\ i_{n} k+j_{n} l & =d_{0}+n \\ j_{n} & <k\end{cases}
$$

where $\left(a_{n}, b_{n}\right)$ is defined by $\left(a_{0}, b_{0}\right)=(0,0)$ and

$$
\begin{aligned}
\left(\begin{array}{l}
a_{n+1} \\
b_{n+1}
\end{array}\right) & =\left(\begin{array}{l}
a_{n} \\
b_{n}
\end{array}\right)+\left(\begin{array}{l}
1 \\
0
\end{array}\right) \text { if } j_{n}-v \geq 0 \\
& =\left(\begin{array}{l}
a_{n} \\
b_{n}
\end{array}\right)+\left(\begin{array}{l}
0 \\
1
\end{array}\right) \text { if } j_{n}-v<0 .
\end{aligned}
$$

Notice that $a_{n}$ is the number of translations of type $(1,1)$ occuring in a path of lenght $n$, and corresponds to the horizontal component of the sum of the $n$ first translations. We consider the left side of the triangle given by the equation

$$
k i-(k-v) j+\nu_{c}(k-v)=0
$$


and its intersections $\left(x_{n}, n\right)$ with the horizontal levels $j=n$. We have

$$
x_{n}=\frac{k-v}{k}\left(n-\nu_{c}\right) \text {. }
$$

We consider the path starting from the last integer point $([c[+1,0)$. The successive integer points of this path are given by the sequence $\left(p_{n}, n\right)=\left(\left[c\left[+1+a_{n}, n\right)\right.\right.$. We claim that the moving point along this path does not go too far away from the left side of the triangle. More precisely, we have:

$$
\left.\left.\left(p_{n}-x_{n}\right) \in\right]-1,1\right]
$$

Indeed, by solving the above system, we obtain

$$
a_{n}=\frac{-j_{n}}{k}+n \frac{k-v}{k} .
$$

Therefore we have:

$$
p_{n}-x_{n}=\left(\left[-\nu_{c} \frac{k-v}{k}\left[+\nu_{c} \frac{k-v}{k}+1\right)+\left(a_{n}-n \frac{k-v}{k}\right) .\right.\right.
$$

Clearly, the first part of the sum belongs to ]0,1], and the second one, which equals to $\frac{-j_{n}}{k}$ belongs to $]-1,0]$. Therefore this path will catch all the first integer points of the triangle on each level starting from the left side. If we consider the $p$ parallel paths starting from $M_{i}, k=1, \cdots p$, they will catch all the integers points of the triangle, since on each level there is at most $p$ points.

These $p$ paths give us a unique way to distribute the monomials $a_{b, d} m^{d}$ on each branch, putting the monomials encountered on the first path (starting from the right hand side) on the first branch, and so on. With this path game, we do not miss any point of the triangle according to the previous proposition. Each integer point of the triangle can be represented by the new coordinates $(b, d)$ ( $b$ : the index of a path or branch; $d$ : the index of a level, or degree). From our construction, they are related to $(i, j)$ by the change of coordinates

$$
(i, j)=\Phi(b, d)=\left(\left[c\left[+p+1-b+\sum_{d=k l-1}^{d-1} \alpha_{d}, d-k l\right),\right.\right.
$$

where $\alpha_{k l-1}=0$, and for $d \geq k l, \alpha_{d}$ is the horizontal component of $\overrightarrow{t_{d}}$.

In conclusion, the general writing of the analytic normal forms for foliations defined by a function in $\mathcal{T}$ obtained by our construction is:

$$
N_{a}=x^{n_{0}} y^{n_{\infty}} \prod_{b=1}^{p}\left(y^{k}+\sum_{\Phi(b, d) \in \mathbb{T}} a_{b, d} m^{d}\right)^{n_{b}}
$$

where $a=\left(a_{b, d}\right)$ belongs to the open set $\mathcal{A}$ of $\mathbb{C}^{\delta}$ defined by $a_{1, k l}=1, a_{b, k l} \neq 0, a_{b, k l} \neq a_{b^{\prime}, k l}$ for $b \neq b^{\prime}$, and $\Phi(b, d)$ is defined by (4).

Example. From the figure (6) in the Appendix C, the analytic normal form $N_{a}$ of the foliation defined by a function $f$ in the topological class $(k, l)=(3,5), p=4, n=$ $\left(n_{1}, n_{2}, n_{3}, n_{4}\right)$ are given in the same Appendix: we add 2 monomials on the first branch, 16 on the second, 31 on the third and 29 on the last one.

Remark 1.9. Contrary to $\vec{x}$ and $\vec{y}$, the translations $\overrightarrow{t_{d}}$ are not well defined in the cohomological module, since they don't keep invariant the half planes corresponding to $\Theta\left(U_{0}\right)$ and $\Theta\left(U_{\infty}\right)$. For this reason, we will only consider their action on the global half plane, i.e. on the set $\Theta_{\mathcal{F}}\left(U_{0} \cap U_{\infty}\right)$ of cocycles. 


\subsection{Local universality}

The construction described in the previous section is justified, a posteriori, by the following result:

Theorem 1.10. We fix $a^{0}=\left(a_{b, d}^{0}\right)$ in $\mathcal{A}$, and we consider the foliation $\mathcal{F}_{0}$ defined by $N_{a^{0}}$. The unfolding $\left\{N_{a}, a \in\left(\mathcal{A}, a^{0}\right)\right\}$ is a semi-universal one among the equireducible unfoldings of $\mathcal{F}_{0}$.

This means that for any equireducible unfolding $\left\{F_{t}, t \in\left(T, t^{0}\right)\right\}$ which defines $\mathcal{F}_{0}$ for $t=t^{0}$, there exists a map $\lambda: T \rightarrow A$ such that the family $F_{t}$ is analytically equivalent to $N_{\lambda(t)}$. Furthermore, the universality means that the map $\lambda$ is unique and the semiuniversality only requires that the first derivative of $\lambda$ at $t^{0}$ is unique.

Proof - Let $E$ be the common desingularization map for each normal form $N_{a}$ and $\widetilde{\mathcal{F}_{a}}$ the foliation defined by $\widetilde{N_{a}}=N_{a} \circ E$. Let $\Theta_{0}$ be the sheaf on $D$ of germs of holomorphic vector fields tangent to the foliation $\widetilde{\mathcal{F}}_{a^{0}}$. We consider the unfolding $\left\{\widetilde{\mathcal{F}}_{a}, a \in\left(\mathcal{A}, a^{0}\right)\right\}$ of $\widetilde{\mathcal{F}_{a^{0}}}$.

Lemma 1.11. The unfolding $\left\{\widetilde{\mathcal{F}}_{a}, a \in\left(\mathcal{A}, a^{0}\right)\right\}$ is locally analytically trivial on each open set $U_{0}, U_{\infty}$.

Proof. One can locally solve the equation with the unknown variable $\theta$ :

$$
\theta\left(\widetilde{N_{a}}\right)=\frac{\partial \widetilde{N_{a}}}{\partial a_{b, d}} .
$$

This is clear around a regular point of the foliation, and still true around a reduced singular point: see [12]. Now, from Proposition (3.6) in Appendix A, we can solve this equation globally on $U_{0}$ and $U_{\infty}$. The vector field $-\theta+\frac{\partial}{\partial a_{b, d}}$ is in the kernel of $d \widetilde{N}_{a}$, and its flow defines a local trivialization of this unfolding.

Let $\theta_{b, d}^{0}$ (resp. $\theta_{b, d}^{\infty}$ ) a solution of the above equation in the open set $U_{0}$ (resp. $\left.U_{\infty}\right)$. The cocycle $\left(\theta_{b, d}^{0}-\theta_{b, d}^{\infty}\right)$ defines a unique element of $H^{1}\left(D, \Theta_{0}\right)$ which only depends on the analytic class of the unfolding $\widetilde{\mathcal{F}}_{a}$. We denote it : $\left[\frac{\partial \mathcal{F}_{a}}{\partial a_{b, d}}\right]_{a^{0}}{ }^{2}$. We have defined a map from the tangent space to $\mathcal{A}$ in $a^{0}$ into $H^{1}\left(D, \Theta_{0}\right)$. According to a theorem of J.F. Mattei ([12], theorem (3.2.1)), we have to prove that this map is a bijective one. By our construction, the dimension of $\mathcal{A}$ is equal to $\delta$. Therefore it suffices to prove that the $\delta$ cocycles $\left[\frac{\partial \mathcal{F}_{a}}{\partial a_{b, d}}\right]$ are independent in $H^{1}\left(D, \Theta_{0}\right)$. We denote by

$$
\left\langle\left[\frac{\partial \mathcal{F}_{a}}{\partial a_{b, d}}\right], e_{i, j}\right\rangle
$$

the component of $\left[\frac{\partial \mathcal{F}_{a}}{\partial a_{b, d}}\right]$ on each element of the basis $\left\{e_{i, j}\right\}$ represented by the integer points in the triangular representation of $H^{1}\left(D, \Theta_{0}\right)$. These numbers define a $\delta \times \delta$ matrix $M$, and we have to prove that it is an invertible one, that will be done in several steps.

Step 1. Components of the "Bézout cocycles" of the first level.

We will only compute the cocycles $\left(\theta_{b, d}^{0}-\theta_{b, d}^{\infty}\right)$ in the two charts covering the principal component $D_{c}$. Therefore, for sake of simplicity, we renumber these two charts:

$$
\left(x_{0}, y_{0}\right):=\left(x_{c-1}, y_{c-1}\right) \text { in the notations of Appendix A: chart around }\left(D_{c}, 0\right) \text {; }
$$

\footnotetext{
${ }^{2}$ Since the point $a^{0}$ is fixed, we will frequently omit it in this notation.
} 
$\left(x_{\infty}, y_{\infty}\right):=\left(x_{c}, y_{c}\right)$ in the notations of Appendix A: chart around $\left(D_{c}, \infty\right)$.

We will make use of the quasi-homogeneous degree related to $R=k x \frac{\partial}{\partial x}+l y \frac{\partial}{\partial y}$. One can check that the pull back of $R$ is locally given in the previous charts of the principal component by $x_{0} \frac{\partial}{\partial x_{0}}$ in the chart around $\left(D_{c}, 0\right)$ or by $y_{\infty} \frac{\partial}{\partial y_{\infty}}$ in the chart around $\left(D_{c}, \infty\right)$. Therefore the corresponding graduation is defined by the partial degree in $x_{0}$ in the first chart, and by the partial degree in $y_{\infty}$ in the second one. In what follows, " $R$-degree" refers to this graduation.

Among the solutions of (6) on each open set $U_{0}$ (resp. $U_{\infty}$ ), we choose a particular one, namely the "Bézout solution" caracterized by Bézout conditions: indeed, if we want to find the two components of $\theta_{b, d}^{0}$ satifying (6) solving degree by degree, we find a Bézout identity with polynomial expressions in $y_{0}$, or in $x_{\infty}$ in the other chart. We choose the unique Bézout solution defined by the usual majorations of degrees. In order to precise this, we compute the first term of the Bézout solution for the cocycle related to directions $\frac{\partial}{\partial a_{b, k l}}\left(\right.$ i.e. on the first level $d_{0}=k l$ ). According to Proposition (3.1) in Appendix A, we have:

$$
\begin{aligned}
\widetilde{N_{a}}\left(x_{0}, y_{0}\right) & =x_{0}^{n_{\infty} k+n_{0} l+|n| k l} y_{0}^{n_{\infty} v+n_{0} u+|n| v l} \prod_{b=1}^{p}\left(a_{b, k l}+y_{0}+\cdots\right)^{n_{b}} \\
& =x_{0}^{m_{0}} P\left(y_{0}\right)+\cdots
\end{aligned}
$$

where $|n|=\sum_{b=1}^{p} n_{b}, m_{0}=n_{0} l+n_{\infty} k+|n| k l, P$ is a one variable polynomial, and the dots stand for terms of $R$-degree $d$ higher than $k l$. Therefore we have:

$$
\frac{\partial \widetilde{N_{a}}}{\partial x_{0}}=m_{0} x_{0}^{m_{0}-1} P\left(y_{0}\right)+\cdots, \quad \frac{\partial \widetilde{N_{a}}}{\partial y_{0}}=x_{0}^{m_{0}} P^{\prime}\left(y_{0}\right)+\cdots
$$

Now we have

$$
\frac{\partial \widetilde{N}_{a} / \partial a_{b, k l}}{\widetilde{N}_{a}}=\frac{n_{b}}{a_{b, k l}+y_{0}}+\cdots
$$

and therefore

$$
\frac{\partial \widetilde{N_{a}}}{\partial a_{b, k l}}=\frac{n_{b} x_{0}^{m_{0}} P\left(y_{0}\right)}{a_{b, k l}+y_{0}}+\cdots
$$

Setting $\theta_{b, k l}^{0}=m_{0}^{-1} x_{0} \alpha_{b, k l}^{0}\left(y_{0}\right) \frac{\partial}{\partial x_{0}}+\beta_{b, k l}^{0}\left(y_{0}\right) \frac{\partial}{\partial y_{0}}+\cdots$ and identifying the terms of lower $R$-degree in equation (6) on $U_{0}$, we obtain

$$
\alpha_{b, k l}^{0} P+\beta_{b, k l}^{0} P^{\prime}=\frac{n_{b} P}{a_{b, k l}+y_{0}} .
$$

We consider the unique solution $\left(U_{0}, V_{0}\right)$ of the following Bézout identity in $\mathbb{C}\left[y_{0}\right]$ :

$$
U_{0} P+V_{0} P^{\prime}=P \wedge P^{\prime}, \operatorname{deg}\left(U_{0}\right)<\operatorname{deg}\left(P^{\prime} / P \wedge P^{\prime}\right), \operatorname{deg}\left(V_{0}\right)<\operatorname{deg}\left(P / P \wedge P^{\prime}\right),
$$

Notice that $\operatorname{deg}\left(P / P \wedge P^{\prime}\right)=p+1$. We obtain an holomorphic solution $\theta_{b, k l}^{0}$ of (6) at the first order by setting

$$
\alpha_{b, k l}^{0}=\frac{n_{b} U_{0} P}{\left(P \wedge P^{\prime}\right)\left(a_{b, k l}+y_{0}\right)}, \quad \beta_{b, k l}^{0}=\frac{n_{b} V_{0} P}{\left(P \wedge P^{\prime}\right)\left(a_{b, k l}+y_{0}\right)} .
$$

We can perform a similar computation in the other chart $\left(x_{\infty}, y_{\infty}\right)$ on $U_{\infty}$. We have:

$$
\begin{aligned}
\widetilde{N}_{a}\left(x_{\infty}, y_{\infty}\right) & =x_{\infty}^{k n_{0}-v n_{0}+l n_{\infty}-u n_{\infty}+|n|(k l-k u)} y_{\infty}^{k n_{0}+l n_{\infty}+|n| k l} \prod_{b=1}^{p}\left(1+a_{b, k l} x_{\infty}+\cdots\right)^{n_{b}} \\
& =y_{\infty}^{m_{0}} Q\left(x_{\infty}\right)+\cdots
\end{aligned}
$$


Setting $\theta_{b, k l}^{\infty}=\alpha_{b, k l}^{\infty}\left(x_{\infty}\right) \frac{\partial}{\partial x_{\infty}}+m_{0}^{-1} y_{\infty} \beta_{b, k l}^{\infty}\left(x_{\infty}\right) \frac{\partial}{\partial y_{\infty}}+\cdots$, we have to solve

$$
\alpha_{\infty} Q^{\prime}+m_{0} \beta_{\infty} Q=\frac{n_{b} x_{\infty} Q}{1+a_{b, k l} x_{\infty}}
$$

By considering the solution $\left(U_{\infty}, V_{\infty}\right)$ of the following Bézout identity:

$$
U_{\infty} Q+V_{\infty} Q^{\prime}=Q \wedge Q^{\prime}, \operatorname{deg}\left(U_{\infty}\right)<\operatorname{deg}\left(\frac{Q^{\prime}}{Q \wedge Q^{\prime}}\right), \operatorname{deg}\left(V_{\infty}\right)<\operatorname{deg}\left(\frac{Q}{Q \wedge Q^{\prime}}\right)
$$

we obtain a holomorphic solution at first order of (6) on $U_{\infty}$ by setting:

$$
\alpha_{b, k l}^{\infty}=\frac{n_{b} x_{\infty} Q V_{\infty}}{\left(1+a_{b, k l} x_{\infty}\right)\left(Q \wedge Q^{\prime}\right)}, \beta_{b, k l}^{\infty}=\frac{n_{b} x_{\infty} Q U_{\infty}}{\left(1+a_{b, k l} x_{\infty}\right)\left(Q \wedge Q^{\prime}\right)} .
$$

We can compute now the first term of the Bézout cocycles related to the directions $\frac{\partial}{\partial a_{b, k l}}$. According to our construction of the normal forms, the coefficient $a_{1, k l}$ is constant equal to 1 . Nevertheless, in order to perform calculus in a more symmetric way, we first consider the unfolding in which $a_{1, k l}$ is a free parameter. In this unfolding, $a_{b, k l}$ are only invariants up to a multiplicative constant. Then, we will set $a_{1, k l}=1$.

We denote by $\frac{\partial \mathcal{F}_{a}}{\partial a_{b, d}}$ (without brakets) the Bézout cocycle $\left(\theta_{b, k l}^{0}-\theta_{b, k l}^{\infty}\right)$ in $\Theta_{0}\left(U_{0} \cap U_{\infty}\right)$, and $\left[\frac{\partial \mathcal{F}_{a}}{\partial a_{b, d}}\right]$ (in brakets) its image in $H^{1}\left(D, \Theta_{0}\right)$.

Lemma 1.12. The matrix defined by the components of the $p$ Bézout cocycles $\frac{\partial \mathcal{F}_{a}}{\partial a_{b, k l}}$ in $\Theta_{0}\left(U_{0} \cap U_{\infty}\right)$, on $e_{\Phi\left(b^{\prime}, k l\right)}, b=1, \ldots p, b^{\prime}=1, \ldots p$, is an invertible $p \times p$ Vandermonde matrix $V$.

Proof. In order to compute the Bézout cocycles, we give the expression of $\theta_{b, k l}^{0}$ in the chart $\left(x_{\infty}, y_{\infty}\right)$. Since we have $x_{0}=x_{\infty} y_{\infty}, \quad y_{0}=x_{\infty}^{-1}$, we obtain

$$
\frac{\partial}{\partial x_{0}}=x_{\infty}^{-1} \frac{\partial}{\partial y_{\infty}}, \quad \frac{\partial}{\partial y_{0}}=-x_{\infty}^{2} \frac{\partial}{\partial x_{\infty}}+x_{\infty} y_{\infty} \frac{\partial}{\partial y_{\infty}}
$$

Furthermore, by considering the reduced polynomials related to $P$ and $Q$, we also have

$$
\frac{P}{P \wedge P^{\prime}}\left(y_{0}\right)=\frac{1}{x_{\infty}^{p+2}} \frac{Q}{Q \wedge Q^{\prime}}\left(x_{\infty}\right) .
$$

We obtain:

$\theta_{b, k l}^{0}=\frac{n_{b} x_{\infty}^{-(p+2)} Q / Q \wedge Q^{\prime}\left(x_{\infty}\right)}{\left(a_{b, k l}+x_{\infty}^{-1}\right)}\left[m_{0}^{-1} U_{0}\left(x_{\infty}^{-1}\right) y_{\infty} \frac{\partial}{\partial y_{\infty}}+V_{0}\left(x_{\infty}^{-1}\right)\left(-x_{\infty}^{2} \frac{\partial}{\partial x_{\infty}}+x_{\infty} y_{\infty} \frac{\partial}{\partial y_{\infty}}\right)\right]$

We consider now a vector field $\theta_{i s}$ on $U_{0} \cap U_{\infty}$ tangent to the foliation defined by $\widetilde{N_{a}}$, with isolated singularities. Since

$$
\left(-\frac{\partial \widetilde{N_{a}}}{\partial y_{\infty}}, \frac{\partial \widetilde{N_{a}}}{\partial x_{\infty}}\right)=\left(-m_{0} y_{\infty}^{m_{0}-1} Q\left(x_{\infty}\right)+\cdots, y_{\infty}^{m_{0}} Q^{\prime}\left(x_{\infty}\right)+\cdots\right)
$$

we can choose

$$
\theta_{i s}=\left(-\frac{Q}{Q \wedge Q^{\prime}}+\cdots\right) \frac{\partial}{\partial x_{\infty}}+\left(y_{\infty} \frac{Q^{\prime}}{m_{0} Q \wedge Q^{\prime}}+\cdots\right) \frac{\partial}{\partial y_{\infty}} .
$$


Let $\Phi_{b, k l}^{0, \infty}$ the coefficient such that $\theta_{b, k l}^{0}-\theta_{b, k l}^{\infty}=\Phi_{b, k l}^{0, \infty} \cdot \theta_{i s}$. By computing the coefficient of $\theta_{b, k l}^{0}-\theta_{b, k l}^{\infty}$ on $\partial / \partial x_{\infty}$, we have:

$$
\Phi_{b, k l}^{0, \infty}=\frac{n_{b} x_{\infty}}{1+a_{b, k l} x_{\infty}}\left[x_{\infty}^{-p} V_{0}\left(x_{\infty}^{-1}\right)-V_{\infty}\left(x_{\infty}\right)\right] .
$$

The components of $\frac{\partial \mathcal{F}_{a}}{\partial a_{b, k l}}$ on $e_{\Phi\left(b^{\prime}, k l\right)}$, i.e. on $e_{i, 0}, i=[c[+p,[c[+p-1, \ldots[c[+1$, are the coefficients on $x_{\infty}^{-i}$ of the Laurent series of $\Phi_{b, k l}^{0, \infty}$. We only have to consider the meromorphic part of $\Phi_{b, k l}^{0, \infty}$, i.e.:

$$
\frac{n_{b} x_{\infty}}{\left(1+a_{b, k l} x_{\infty}\right)} \times \frac{\overline{V_{0}}\left(x_{\infty}\right)}{x_{\infty}^{2 p}}
$$

where $\overline{V_{0}}(x)=\sum_{n=0}^{p} v_{n} x^{n}$ is the polynomial $x^{p} V_{0}\left(x^{-1}\right)\left(\right.$ recall that $\left.\operatorname{deg}\left(V_{0}\right) \leq p\right)$. We have

$$
\begin{aligned}
\frac{x_{\infty}}{1+a_{b, k l} x_{\infty}} & =\sum_{m=0}^{+\infty}\left(-a_{b, k l}\right)^{m} x_{\infty}^{m+1} \\
\frac{\overline{V_{0}}\left(x_{\infty}\right)}{x_{\infty}^{2 p}} & =\sum_{n=0}^{p} v_{n} x_{\infty}^{n-2 p} .
\end{aligned}
$$

Therefore, the coefficient of the Laurent series of $\Phi_{0, \infty}$ in $x_{\infty}^{-i}$ is

$$
\begin{aligned}
\sum_{(m+1)+(n-2 p)=-i} n_{b} v_{n}\left(-a_{b, k l}\right)^{m} & =n_{b} \sum_{n=0}^{p} v_{n}\left(-a_{b, k l}\right)^{2 p-1-n-i} \\
& =n_{b} \overline{V_{0}}\left(-a_{b, k l}^{-1}\right) \times\left(-a_{b, k l}\right)^{2 p-1-i} \\
& =n_{b} \overline{V_{0}}\left(-a_{b, k l}^{-1}\right) \times\left(-a_{b, k l}\right)^{(p-2-[c])+b^{\prime}}
\end{aligned}
$$

since, from (4), we have $i=[c[+p+1-b$. Finally we obtain

$$
<\frac{\partial \mathcal{F}_{a}}{\partial a_{b, k l}}, e_{\Phi\left(b^{\prime}, k l\right.}>=\lambda_{b}\left(\mu_{b}\right)^{b^{\prime}}, \text { with } \mu_{b}=-a_{b, k l},
$$

which defines a Vandermonde matrix. Furthermore, $\mu_{b} \neq 0,1$ and the coefficients $\lambda_{b}$ do not vanish. Indeed, $\lambda_{b}=0$ if and only if $\overline{V_{0}}\left(-a_{b, k l}^{-1}\right)=0$, i.e. $V_{0}\left(-a_{b, k l}\right)=0$. Evaluating the Bézout identity

$$
U_{0} \frac{P}{P \wedge P^{\prime}}+V_{0} \frac{P^{\prime}}{P \wedge P^{\prime}}=1
$$

at $y_{0}=-a_{b, k l}$, we would obtain a contradiction, since $-a_{b, k l}$ is a root of $P$. Therefore, the determinant of $V$ doesn't vanish.

Step 2. Components of the Bézout cocycles of higher degree.

Lemma 1.13. 1) The components $\left\langle\frac{\partial \mathcal{F}_{a}}{\partial a_{b, d}}, e_{i, j}\right\rangle$ of the Bézout cocycles of higher levels vanish on the strictly lower levels $j<d-k l$.

2) Let us consider the decomposition of $\left[\frac{\partial}{\partial a_{b, d}}\right]$ in the basis $e_{i, j}$ :

$$
\left[\frac{\partial}{\partial a_{b, d}}\right]=\sum_{j \geq d-k l} \sum_{i} A_{i, j}^{b, d}(a) e_{i, j}
$$

The function $A_{i, j}^{b, d}(a)$ only depends on the variables $a_{b, d^{\prime}}$ with $0 \leq d^{\prime}-k l \leq j-(d-k l)$. 
Proof. 1) Using the logarithmic derivatives, we check that the equation $\theta\left(\widetilde{N_{a}}\right)=\frac{\partial \widetilde{N_{a}}}{\partial a_{b, d}}$ is equivalent to

$$
\begin{aligned}
\sum_{b^{\prime}=1}^{p} n_{b^{\prime}} & \theta\left(\widetilde{B_{b^{\prime}}}\right) \\
\widetilde{B_{b^{\prime}}} & =n_{b} \frac{\widetilde{m^{d}}}{\widetilde{B_{b}}} . \\
m^{k l} & =\widetilde{x^{l}}=x_{\infty}^{k l-v l} y_{\infty}^{k l} ; \\
\widetilde{B_{b}} & =x_{\infty}^{k l-k u} y_{\infty}^{k l}+a_{b, k l} x_{\infty}^{k l-v l} y_{\infty}^{k l}+\cdots
\end{aligned}
$$

where the dots stand for terms of degree $d$ higher than $k l$. Therefore, for $d=k l$ we have

$$
n_{b} \frac{\widetilde{m^{k l}}}{\widetilde{B_{b}}}=n_{b} \frac{x_{\infty}}{1+a_{b, d} x_{\infty}}+\cdots=n_{b} \frac{1}{y_{0}+a_{b, d}}+\cdots
$$

and for $d>k l$

$$
n_{b} \frac{\widetilde{m^{d}}}{\widetilde{B_{b}}}=n_{b} \frac{x_{\infty} y_{\infty}^{d-k l}}{1+a_{b, d} x_{\infty}}+\cdots=n_{b} \frac{x_{0}^{d-k l}}{y_{0}+a_{b, d}}+\cdots
$$

Therefore the components of the cocycle on $e_{i, j}$ vanish for $j<d-k l$.

2) The function $A_{i, j}^{b, d}(a)$ is the coefficient of $x_{\infty}^{i} y_{\infty}^{j}$ in the Laurent developpement of the function $\Phi_{b, d}^{0, \infty}$ which is defined by the relation

$$
\theta_{b, d}^{0}-\theta_{b, d}^{\infty}=\Phi_{b, d}^{0, \infty} \theta_{i s} .
$$

Now, the vector fields $\theta_{b, d}^{0}$ and $\theta_{b, d}^{\infty}$ are defined on their respective open set of definition by the relation

$$
\theta_{b, d}^{\star}\left(\widetilde{N_{a}}\right)=\frac{\partial \widetilde{N_{a}}}{\partial a_{b, d}}
$$

Let us filter the above relation with respect to $y_{\infty}$-valuation

$$
\sum_{N} \sum_{p+q=N} \theta_{b, d}^{\star,(p)}\left(\widetilde{N}_{a}^{(q)}\right)=\sum_{N}\left(\frac{\partial \widetilde{N_{a}}}{\partial a_{b, d}}\right)^{(N)} .
$$

In this decomposition, the vector field $\theta_{b, d}^{\star,(p)}$ is written $(\cdot) \frac{\partial}{\partial x_{\infty}}+(\cdot) y_{\infty} \frac{\partial}{\partial y_{\infty}}$ where the coefficients have a $y_{\infty}$-valuation equal to $p$. Thus, the term $\theta_{b, d}^{\star,(p)}\left({\widetilde{N_{a}}}^{(q)}\right)$ has exactly a $y_{\infty}$ valuation equal to $N$. Let $q_{0}$ be the smallest integer such that $\widetilde{N}_{a}^{\left(q_{0}\right)} \neq 0$. We know that $\theta_{b, d}^{\star,(p)}=0$ if $p \leq d-k l$. Thus, the equation yields

$$
\sum_{\substack { p+q=N \\
\begin{subarray}{c}{q \geq q_{0} \\
p \geq d-k l{ p + q = N \\
\begin{subarray} { c } { q \geq q _ { 0 } \\
p \geq d - k l } }\end{subarray}} \theta_{b, d}^{\star,(p)}\left(\widetilde{N}_{a}^{(q)}\right)=\left(\frac{\partial \widetilde{N_{a}}}{\partial a_{b, d}}\right)^{(N)} .
$$


Then, the vector field $\theta_{b, d}^{\star,(n)}$ are inductively defined by

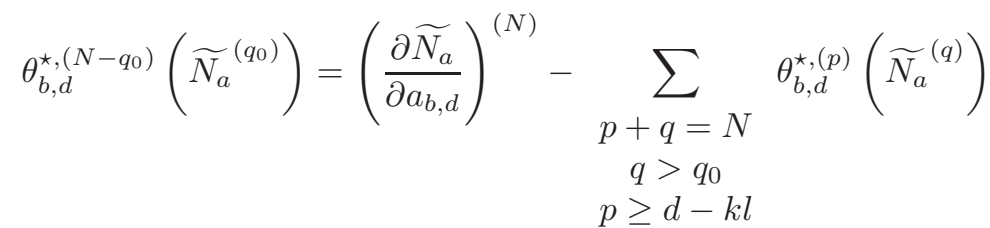

We prove inductively that $\theta_{b, d}^{\star,(n)}$ depends only on $a_{b, d^{\prime}}$ with $0 \leq d^{\prime}-k l \leq n-(d-k l)$. This is already known for $n=d-k l$. Now, suppose this is true until $n=\bar{N}-q_{0}-1$. Let us prove it for $n=N-q_{0}$.

- The vector field $\theta_{b, d}^{\star,(p)}$ depends only on $a_{b, d^{\prime}}$ with $0 \leq d^{\prime}-k l \leq p-(d-k l)<$ $N-q_{0}-(d-k l)=n-(d-k l)$

- If the variables $a_{b, d^{\prime}}$ appears in $\widetilde{N}_{a}^{(q)}$ then $d^{\prime}+q_{0}-k l \leq q$ therefore $d^{\prime}-k l \leq q-q_{0} \leq$ $N-d+k l-q_{0}=n-(d-k l)$

- Finally, if $a_{b, d^{\prime}}$ appears in $\left(\frac{\partial \widetilde{N_{a}}}{\partial a_{b, d}}\right)^{(N)}$ then $d^{\prime}-k l+d-k l+q_{0} \leq N$ which means that $d^{\prime}-k l \leq n-(d-k l)$.

Thus the lemma is proved.

Step 3. Relationship between the Bézout cocycles of different levels.

Lemma 1.14. 1. Let $\overrightarrow{t_{d}}$ the translation introduced at the step 3 of the construction of normal forms, acting on the Bézout cocycles. We have:

$$
\overrightarrow{t_{d}} \cdot \frac{\partial \mathcal{F}_{a}}{\partial a_{b, d}}=\frac{\partial \mathcal{F}_{a}}{\partial a_{b, d+1}} .
$$

2. For each $b$, $b^{\prime}$ in $\{1, \cdots p\}$, the value $<\frac{\partial \mathcal{F}_{a}}{\partial a_{b, d}}, e_{\Phi\left(b^{\prime}, d\right)}>$ is constant with respect to $d$.

Proof. 1. If $\left(\theta_{b, d}^{0}, \theta_{b, d}^{\infty}\right)$ is a solution of $(8)$ on $\left(U_{0}, U_{\infty}\right)$ for the value $(b, d)$, then multiplying this solution by the meromorphic monomial term $\frac{m^{d+1}}{m^{d}}$ yields a solution of $(8)$ on $\left(U_{0}, U_{\infty}\right)$ for the index $(b, d+1)$. This solution is still holomorphic on each open set. Indeed, in the chart $\left(x_{c}, y_{c}\right)$, either $\frac{m^{d+1}}{m^{d}}=x_{c} y_{c}$ or $\frac{m^{d+1}}{m^{d}}=y_{c}$. Therefore, the solution is still holomorphic on $V_{c} \subset U_{\infty}$. Now, $\theta_{\infty}$ has an holomorphic extension on $U_{\infty}$, and one can check, using the changes of charts of Appendix A, that $y_{c}$ or $x_{c} y_{c}$ has also an holomorphic extension on $U_{\infty}$. Indeed,

$$
\begin{aligned}
y_{c} & =x_{c+1} y_{c+1}^{e_{c+1}}=y_{c+2}^{-1}\left(x_{c+2} y_{c+2}^{e_{c+2}}\right)^{e_{c+1}}=\text { etc... } \\
x_{c} y_{c} & =x_{c+1} y_{c+1}^{e_{c+1}-1}=y_{c+2}^{-1}\left(x_{c+2} y_{c+2}^{e_{c+2}}\right)^{e_{c+1}-1}=\text { etc... }
\end{aligned}
$$

and the holomorphy of these extensions comes from $e_{i} \geq 2$ for any $i=c+1, \cdots N$. We have a similar argument on $U_{0}$.

2. On the otherside, the definition of the paths and of the map $\Phi$ in the step 3 of the construction of normal forms yields to $\overrightarrow{t_{d}} \cdot e_{\Phi(b, d)}=e_{\Phi(b, d+1)}$. Therefore we have:

$$
\begin{aligned}
\frac{\partial \mathcal{F}_{a}}{\partial a_{b, d+1}}=\overrightarrow{t_{d}} \cdot \frac{\partial \mathcal{F}_{a}}{\partial a_{b, d}} & =\sum_{b^{\prime}}<\frac{\partial \mathcal{F}_{a}}{\partial a_{b, d}}, e_{\Phi\left(b^{\prime}, d\right)}>\overrightarrow{t_{d}} \cdot e_{\Phi\left(b^{\prime}, d\right)} \\
& =\sum_{b^{\prime}}<\frac{\partial \mathcal{F}_{a}}{\partial a_{b, d}}, e_{\Phi\left(b^{\prime}, d\right)}>e_{\Phi\left(b^{\prime}, d+1\right)}
\end{aligned}
$$

which proves the second part of the lemma. 
End of the proof of Theorem (1.10). From Lemma (1.13), we obtain that the $\delta \times \delta$ matrix

$$
M=\left\langle\left[\frac{\partial \mathcal{F}_{a}}{\partial a_{b, d}}\right], e_{\Phi(b, d)}\right\rangle
$$

is an upper block triangular matrix. Each diagonal block is related to a level $d$ of the triangle $\mathbb{T}$ and its size $n_{d}$ is the number of integer points on this line in $\mathbb{T}$.

The first $(p-1) \times(p-1)$ block can be deduced from Lemma (1.12) by considering the cohomological classes $\left[\frac{\partial \mathcal{F}_{a}}{\partial a_{b, k l}}\right]$ related to each Bézout cocycle. Since $a_{1, k l}=1$, we delete the first column, and since $\left[e_{[c[+p, 0}\right]=0$-the first line has only $p-1$ integer points in the triangle--, we delete the first line. We obtain a $(p-1) \times(p-1)$ Vandermonde sub-matrix of $V$, which is still invertible.

From Lemma (1.14), the next diagonal blocks are also sub-matrices of consecutive lines and columns of $V$. Therefore, they are also Vandermonde non trivial matrices.

Finally, $M$ is an upper block triangular matrix such that each diagonal block is a Vandermonde sub-matrix of $V$. Therefore $M$ is an invertible matrix.

\subsection{The global moduli space of foliations.}

In this section, we are going to use the Theorem (1.10), that is to say, the infinitesimal existence and unicity of a normal form for any function near $N_{a_{0}}$ to get a global statement.

Proposition 1.15 (Existence of normal forms). For any $f$ in $\mathcal{T}$, there exists a in $\mathcal{A}$ such that $f \sim N_{a}$.

Proof. There exists some coordinates $(x, y)$ such that

$$
f=u(x, y) x^{n_{0}} y^{n_{\infty}} \prod_{b=1}^{p}\left(y^{k}+a_{b, k l} x^{l}+p_{b}(x, y)\right)^{n_{b}}
$$

where $u(x, y)$ is a unity and $p_{b}(x, y)$ has a $(k, l)$-valuation greater than $k l$. Following a result of finite determinacy due to Mather [10], we can suppose that $u$ and $p_{b}$ are polynomials. Hence, we can decompose the functions $u$ and $p_{b}$ in $(k, l)$-homogeneous components

$$
f=\left(1+\sum_{\nu \geq 1}^{N} u_{\nu}\right) x^{n_{0}} y^{n_{\infty}} \prod_{b=1}^{p}\left(y^{k}-a_{b, k l} x^{l}+\sum_{\nu \geq k l+1}^{N_{b}} p_{b, \nu}\right)^{n_{b}} .
$$

Let us consider the deformation defined by the following parametrized family of functions $f_{\left(u_{i, j}\right),\left(p_{i, j, b}\right)}$

$$
(1+\sum_{\nu \geq 1}^{N} \underbrace{\sum_{k i+l j=\nu} u_{i, j} x^{i} y^{j}}_{u_{\nu}}) x^{n_{0}} y^{n_{\infty}} \prod_{b=1}^{p}\left(y^{k}-a_{b, k l} x^{l}+\sum_{\nu \geq k l+1}^{\sum_{b}^{N_{b}} \underbrace{\sum_{k i+l j=\nu} p_{i, j, b} x^{i} y^{j}}_{p_{b, \nu}}}\right)^{n_{b}} .
$$

where the set of parameters $\left(u_{i, j}\right)$ and $\left(p_{i, j, b}\right)$ are the coefficients of the polynomials function $u_{\nu}$ and $p_{b, \nu}$. If we set $\left(u_{i, j}\right)=0$ and $\left(p_{i, j, b}\right)=0$ then

$$
f_{(0),(0)}=x^{n_{0}} y^{n_{\infty}} \prod_{b=1}^{p}\left(y^{k}-a_{b, k l} x^{l}\right)^{n_{b}}=N_{a^{0}}
$$


where $a_{0}=\left(a_{b, k l}, 0, \cdots 0\right)$. Now we have the following relation: for any $\lambda \in \mathbb{C}^{*}$

$$
f_{\left(u_{i, j}\right),\left(p_{i, j, b}\right)}\left(\lambda^{k} x, \lambda^{l} y\right)=\lambda^{r} f_{\lambda\left(u_{i, j}\right), \lambda\left(p_{i, j, b}\right)}
$$

where

$$
\begin{aligned}
\lambda\left(u_{i, j}\right) & =\left(\lambda^{k i+l j-k l} u_{i, j}\right) \\
\lambda\left(p_{i, j, b}\right) & =\left(\lambda^{k i+l j-k l} p_{i, j, b}\right)
\end{aligned}
$$

and $r=k n_{0}+l n_{\infty}+k l \sum n_{b}$. The equation (10) ensures that $f_{\lambda\left(u_{i, j}\right), \lambda\left(p_{i, j, b}\right)} \sim f_{\left(u_{i, j}\right),\left(p_{i, j, b}\right)}$. Applying Theorem(1.10) to $f_{(0),(0)}=N_{a^{0}}$ shows that for $\left(u_{i, j}\right),\left(p_{i, j, b}\right)$ small enough,

$$
f_{\left(u_{i, j}\right),\left(p_{i, j, b}\right)} \sim N_{a}
$$

for some $a$ in a neighborhood of $a^{0}$ in $\mathcal{A}$. Now, when $\lambda$ goes to zero, $f_{\lambda\left(u_{i, j}\right), \lambda\left(p_{i, j, b}\right)}$ goes to $f_{(0),(0)}$. Hence, for $\lambda$ small enough and for some $a$ in $\mathcal{A}$ we have

$$
N_{a} \sim f_{\lambda\left(u_{i, j}\right), \lambda\left(p_{i, j, b}\right)} \sim f_{\left(u_{i, j}\right),\left(p_{i, j, b}\right)},
$$

which proves the proposition.

Let us consider the diffeomorphism: $h_{\lambda}(x, y)=\left(\lambda^{k} x, \lambda^{l} y\right)$. We have:

$$
N_{a} \circ h_{\lambda}=\lambda^{|n|} N_{\lambda \cdot a}, \quad \text { with } \lambda \cdot a=\lambda \cdot\left(a_{b, d}\right):=\left(\lambda^{d-k l} a_{b, d}\right) \text { and }|n|=\sum_{b} n_{b} .
$$

As above, we have thus $N_{a} \sim N_{\lambda \cdot a}$. Actually, this action of $\mathbb{C}^{*}$ the only obstruction to the unicity of normal forms:

Theorem 1.16 (Unicity of normal forms). $N_{a} \sim N_{a^{\prime}}$ if and only there exists a complex number $\lambda \neq 0$ such that $a^{\prime}=\lambda \cdot a$.

Proof. Step 1 - Preparation of the conjugacy - Suppose that there exists a conjugacy relation

$$
\psi \circ N_{a}=N_{a^{\prime}} \circ \phi
$$

Following [1], we can suppose that $\psi$ is an homothetie $\gamma \cdot$ Id. We are going to reduce the proof to the case where $\phi$ is tangent to the identity. In order to do so, we denote by $(\alpha x+\beta y, u x+v y)$ the linear part of $\phi$. Let us take a look at the $(k, l)$-homogeneous part of $N_{a}$ and $N_{a^{\prime}}$ : these are conjugated by the linear part of $\phi$. Hence, we have

$$
\begin{aligned}
& x^{n_{0}} y^{n_{\infty}} \prod_{b=1}^{p}\left(y^{k}-a_{b, k l} x^{l}\right)^{n_{b}}= \\
& \gamma(\alpha x+\beta y)^{n_{0}}(u x+v y)^{n_{\infty}} \prod_{b=1}^{p}\left((u x+v y)^{k}-a_{b, k l}^{\prime}(\alpha x+\beta y)^{l}\right)^{n_{b}} .
\end{aligned}
$$

Looking at the leading coefficient in $x$ and $y$ in the above relation yields respectively to $u=0$ and $\beta=0$. Hence the above equality reduces to

$$
x^{n_{0}} y^{n_{\infty}} \prod_{b=1}^{p}\left(y^{k}-a_{b, k l} x^{l}\right)^{n_{b}}=\gamma \alpha^{n_{0}} v^{n_{\infty}+k \sum_{b \geq 1} n_{b}} x^{n_{0}} y^{n_{\infty}} \prod_{b=1}^{p}\left(y^{k}-a_{b, k l}^{\prime} \frac{\alpha^{l}}{v^{k}} x^{l}\right)^{n_{b}} .
$$

Since the conjugacy preserves the numbering of the branches, for all $b, a_{b, k l}=a_{b, k l}^{\prime} \frac{\alpha^{l}}{v^{k}}$. Since, $a_{1, k l}=a_{1, k l}^{\prime}=1$, we have $\alpha^{l}=v^{k}$ and thus $a_{b, k l}=a_{b, k l}^{\prime}$. Let $\Lambda$ be the linear automorphism

$$
\Lambda(x, y)=\left(\frac{1}{\alpha} x, \frac{1}{v} y\right)=\left(\lambda^{k} x, \lambda^{l} y\right)
$$


for some $\lambda \in \mathbb{C}^{*}$. Then

$$
N_{a^{\prime}} \circ \phi \circ \Lambda=\gamma N_{a} \circ \Lambda=u N_{\lambda \cdot a}
$$

where $u$ stands for some non vanishing number. Since, in the above relation $\phi \circ \Lambda$ is tangent to the identity, it appears that $u=1$. Thus, setting for the sake of simplicity $a=\lambda \cdot a$ and $\phi=\phi \circ \Lambda$ we are led to a relation

$$
N_{a}=N_{a^{\prime}} \circ \phi
$$

where $\phi$ is tangent to the identity.

Step 2 - With a conjugacy tangent to Id - The proof reduces to show that in the situation (11), we have $a=a^{\prime}$. Let us denote by $\mathbb{P}_{\delta}$ the set of quasi-homogeneous polynomial functions of $R$-degree $\delta$. We say that a vector field $\theta$ is quasi-homogeneous of degree $\nu$ if and only if

$$
\theta \cdot \mathbb{P}_{\delta} \subset \mathbb{P}_{\delta+\nu}
$$

Notice that $\nu$ can be negative. For any vector field $\theta$, we make the following decomposition

$$
\theta=\sum_{\nu \geq \nu_{0}} \theta_{\nu}
$$

where $\theta_{\nu}$ is quasi-homogeneous of degree $\nu$. One can easily check the following result

Lemma 1.17. The set of $\nu$ quasi-homogeneous vector fields is spanned by

$$
a_{\nu} x \frac{\partial}{\partial x}, a_{\nu} y \frac{\partial}{\partial y}, x^{n} \frac{\partial}{\partial y}, y^{m} \frac{\partial}{\partial y}
$$

where $a_{\nu}$ is quasi-homogeneous of degree $\nu$ and $n$ and $m$ are defined by $\nu=k n-l$ and $\nu=l m-k$. Notice that both $n$ and $m$ may not exist for a given $\nu$.

Now let $X$ be a germ of formal vector field such that

$$
\phi=e^{X} .
$$

The vector field $X$ can be decomposed in the following way

$$
X=\sum_{\nu \geq \nu_{0}} X_{\nu_{0}}
$$

with $X_{\nu}=a_{\nu} x \frac{\partial}{\partial x}+b_{\nu} y \frac{\partial}{\partial y}+q x^{n} \frac{\partial}{\partial y}+r y^{m} \frac{\partial}{\partial y}$ where $a_{\nu}$ and $b_{\nu}$ are $R$-homogeneous function of degree $\nu$ and $n$ and $m$ defined by $\nu=k n-l$ and $\nu=l m-k$. Notice that $q$ and $r$ may vanish when there is no relations such as $\nu=k n-l$ or $\nu=l m-k$ but also when $\phi$ let invariant one or both axes. Now, suppose that $\nu_{0} \geq p k l$ then following an analogue of the lemma 1.4 in [8], $a=a^{\prime}$. On the contrary, suppose that $\nu_{0}<p k l$. The homogeneous part of smallest degree $N=n_{0} l+n_{\infty} k+k l \sum_{b=1}^{p} n_{b}$ can be written

$$
X_{\nu_{0}} \cdot N_{a}^{(N)}=N_{a}^{\left(N+\nu_{0}\right)}-N_{a^{\prime}}^{\left(N+\nu_{0}\right)}
$$

where $N_{a}^{(q)}$ stands for the $R$-homogeneous part of $N_{a}$ of degree $q$. We will prove that this relation yields to a contradiction with $\nu_{0}<p k l$. After composition with the desingularization map $E$, this relation can be expanded in the chart $\left(x_{0}, y_{0}\right)$ of $\left(D_{c}, 0\right)$ as follows:

$$
\begin{aligned}
& \left(\tilde{a}_{\nu_{0}}+r y_{0}^{u m-v}\right) n_{\infty}+\left(\tilde{b}_{\nu_{0}}+q y_{0}^{v n-u}\right)\left(n_{0}+k \sum_{b=1}^{p} n_{b}\right) \\
& +\underbrace{\left(l \tilde{a}_{\nu_{0}}-k \tilde{b}_{\nu_{0}}-k q y_{0}^{v n-u}+l r y_{0}^{u m-v}\right)}_{R\left(y_{0}\right)} \sum_{b=1}^{p} \frac{n_{b} a_{b, k l}}{y_{0}+a_{b, k l}}=y_{0}^{\delta-v l} \sum_{b=1}^{p} \frac{\lambda_{b}}{y_{0}+a_{b, k l}}
\end{aligned}
$$


where $\lambda_{b}=a_{b, k l+\nu_{0}}-a_{b, k l+\nu_{0}}^{\prime}$. The number $\delta$ in (12) is defined by the relation: $m^{\nu_{0}+k l}=$ $x^{\alpha} y^{\beta}$ and $\delta=\alpha v+\beta u$. Let us denote by $\tilde{Q}\left(y_{0}\right)$ the polynomial function $l \tilde{a}_{\nu_{0}}-k \tilde{b}_{\nu_{0}}$, that is the strict transform of $Q=l a_{\nu_{0}}-k b_{\nu_{0}}$. Let us write

$$
Q=\sum_{\substack{(s, t) \\ s k+t l=\nu_{0}}} r_{s t} x^{s} y^{t} .
$$

Then

$$
\tilde{Q}=\sum_{\substack{(s, t) \\ s k+t l=\nu_{0}}} r_{s t} y^{s v+t u}
$$

The number of monomial terms in $Q$ is in correspondence with the number of positive solutions of $s k+t l=\nu_{0}$. Such a solution $(s, t)$ can be always written

$$
\left\{\begin{array}{l}
s=u \nu_{0}-\Delta l \\
t=-v \nu_{0}+\Delta k
\end{array}\right.
$$

The conditions $s \geq 0$ and $t \geq 0$ reduce to $\frac{v \nu_{0}}{k} \leq \Delta \leq \frac{u \nu_{0}}{l}$. Moreover, the integer $s v+t u$ can be expressed in term of $\Delta$

$$
s v+t u=\left(u \nu_{0}-\Delta l\right) v+\left(-v \nu_{0}+\Delta k\right) u=\Delta(u k-v l)=\Delta .
$$

Hence, the polynomial function $R$ has a very simple form

$$
R=\sum_{\frac{v \nu_{0}}{k} \leq \Delta \leq \frac{u \nu_{0}}{l}} r_{\Delta} y_{0}^{\Delta}-k q y_{0}^{v n-u}+l r y_{0}^{u m-v} .
$$

Notice that $\left|v n-u-\frac{v \nu_{0}}{k}\right|<1$ and $\left|u m-v-\frac{u \nu_{0}}{l}\right|<1$, which ensures that actually $R$ is written

$$
R=\sum_{v n-u \leq \Delta \leq u m-v} r_{\Delta} y_{0}^{\Delta} .
$$

The integer $\delta-v l$ is non negative since

$$
\delta-v l=(\alpha-l) v+\beta u=\frac{\nu_{0}-\beta l}{k} v+\beta u=\frac{v \nu_{0}+\beta}{k} \geq 0 .
$$

Thus, evaluating the residue at $-a_{b, k l}$ in (12) yields the relation

$$
R\left(-a_{b, k l}\right) n_{b} a_{b, k l}=\left(-a_{b, k l}\right)^{\delta-\nu l} \lambda_{b} .
$$

Now, in view of the construction of the normal form, the coefficient $\lambda_{b}$ vanishes for

$$
p-\sharp\left\{-p+\frac{k-v}{k} \nu_{0}+\frac{\varepsilon_{0}}{k}<\delta<\frac{l-u}{l} \nu_{0}-\frac{\varepsilon_{\infty}}{l}\right\}
$$

values of the parameter $b$. Now, we have

$$
\begin{aligned}
& \underbrace{p-\sharp\left\{-p+\frac{k-v}{k} \nu_{0}+\frac{\varepsilon_{0}}{k}<\delta<\frac{l-u}{l} \nu_{0}-\frac{\varepsilon_{\infty}}{l}, \delta \in \mathbb{Z}\right\}}_{\text {number of zeros of } R} \geq \\
& \forall \underbrace{\left\{\frac{v \nu_{0}}{k} \leq \delta \leq \frac{u \nu_{0}}{l}, \delta \in \mathbb{N}\right\}-\varepsilon_{0}-\varepsilon_{\infty}}_{\text {number of monomial terms in } R} .
\end{aligned}
$$


According to the previous inequality, the polynomial function $R$ has to be zero. Therefore, looking again at the relation (12) yields

$$
\forall b, \lambda_{b}=0 .
$$

Hence, the vector field $X_{\nu_{0}}$ has to be tangent to $N_{a}^{(N)}$ which is a contradiction with the hypothesis $\nu_{0}<p k l$.

Finally, we can summarize the previous results by

Theorem 1.18. The moduli space $\mathcal{M}=\mathcal{T} / \sim$ is isomorphic to $\mathcal{A} /(a=\lambda \cdot a)$.

Topological description of $\mathcal{M}$. Notice that the action $\lambda \cdot\left(a_{b, d}\right)=\left(\lambda^{d-k l} a_{b, d}\right)$ keeps invariant each term of the first line $d=k l$. Consider

$$
\mathcal{B}=\left\{a \in \mathcal{A}, a_{b, d}=0 \text { for } d \neq k l\right\} .
$$

Clearly $\mathcal{M}$ is fibered on $\mathcal{B}$, and the fibers are weighted projective spaces on $\mathbb{C}^{\delta-(p-1)}$. Since these fibers are topologically trivial, the topology of $\mathcal{M}$ is completely described by the action of $\pi^{1}(\mathcal{B})$ which is a braid group.

\section{The moduli space of curves.}

Let $\mathcal{C}$ be the partition of $\mathcal{M}$ induced by the classification of curves $\sim_{c}$.

\subsection{The infinitesimal generators of $\mathcal{C}$}

We first recall general facts proved in [8], which are valid in every topological class. Let $\mathcal{F}$ be a foliation defined by an holomorphic function $f$ (or more generally by any generic non dicritical differential form $\omega$ ), and let $S$ be the curve defined by $f=0$ (or by the separatrix set of $\omega)$. Let $E: M \rightarrow\left(\mathbb{C}^{2}, 0\right)$ be the desingularization map of the foliation, and $D$ its exceptional divisor. We denote by $\widetilde{f}, \widetilde{\mathcal{F}}, \widetilde{S}$ the pull back by $E$ on $M$ of $f, \mathcal{F}$ or $S$. The tangent space to the point $[S]$ in the moduli space of curves (for $\sim_{C}$ ) is the cohomological group $H^{1}\left(D, \Theta_{S}\right)$ where $\Theta_{S}$ is the sheaf on $D$ of germs of vector fields tangent to $\widetilde{S}$. The inclusion of $\Theta_{\mathcal{F}}$ into $\Theta_{S}$ induces a map $i$ :

$$
H^{1}\left(D, \Theta_{\mathcal{F}}\right) \stackrel{i}{\longrightarrow} H^{1}\left(D, \Theta_{S}\right)
$$

whose kernel represents the directions of unfolding of foliations with trivial associate unfolding of curves.

Definition 2.1. An open set of $M$ is a quasi-homogeneous open set (relatively to $f$ ) if there exists an holomorphic vector field $R_{U}$ on $U$ such that $R_{U}(\widetilde{f})=\widetilde{f}$.

We can always cover $D$ by quasi-homogeneous open sets. The cocycle of quasi-homogeneity $\left[R_{U, V}\right]$ of $\mathcal{F}$ is the element of $H^{1}\left(D, \Theta_{\mathcal{F}}\right)$ induced by $R_{U}-R_{V}$.

Recall that $H^{1}\left(D, \Theta_{\mathcal{F}}\right)$ has a natural structure of $\mathcal{O}_{2}$-moduli. We have:

Theorem 2.2. [8] The kernel of the map $i$ is generated by the cocycle of quasi-homogeneity, i.e.:

$$
\operatorname{ker}(i)=\left\{h \cdot\left[R_{U, V}\right], h \in \mathcal{O}_{2}\right\} .
$$

Notice that the distribution induced by these directions is integrable and defines a singular foliation $\mathcal{C}$ on $\mathcal{A}$. The point corresponding to the topological model is a singular one: indeed, this model is quasihomogeneous. Therefore the whole open set $U=M$ is quasi-homogeneous, and the cocycle $\left[R_{U, V}\right]$ is trivial for this foliation. 
Let $X_{m, n}$ be the vector fields on $\mathcal{A}$ generated by $x^{m} y^{n} \cdot\left[R_{U, V}\right]$. One can give the explicit expression of $X_{0,0}$ in the coordinates $a_{b, d}$. Indeed, with a very slight modification in the proof of proposition (5.5) of [8] -replace $(\lambda x, \lambda y)$ with $\left(\lambda^{k} x, \lambda^{l} y\right)$-, we obtain:

Proposition 2.3. The $\mathcal{O}_{2}$-generator of $\mathcal{C}$ is given by:

$$
X_{0,0}=-\frac{1}{r} \sum_{\Phi(b, d) \in T}(d-k l) a_{b, d} \frac{\partial}{\partial a_{b, d}} .
$$

where $r=k n_{0}+l n_{\infty}+k l \sum_{b=1}^{p} n_{b}$

In the next result, we give a description of the vector fields $X_{m, n}$. We introduce the following notation for the decomposition of $X_{0,0}$ on the basis $\left\{e_{i, j}\right\}_{(i, j) \in \mathbb{T}}$

$$
X_{0,0}=\sum_{(i, j) \in \mathbb{T}} \Gamma_{i, j}(a) e_{i, j}=\sum_{(b, d)} \Gamma_{\phi(b, d)}(a) e_{\phi(b, d)}
$$

Moreover, for any level $d$ we denote by $X_{m, n}^{d}$ the projection of the vector field $X_{m, n}$ on the subspace $\operatorname{Vect}\left\{e_{\phi(b, d)}, b=1, \cdots p\right\}$.

Proposition 2.4. For any $m, n, X_{m, n}$ is quasihomogeneous with respect to the degree induced by $r X_{0,0}$. Indeed, we have

$$
\left[r X_{0,0}, X_{m, n}\right]=(k m+\ln ) X_{m, n}
$$

The coefficients of $X_{m, n}^{\nu}$ are quasi-homogeneous with respect to the weight $r X_{0,0}$ of degree $\nu-k m-l n-k l$. In particular, they only depend on the variables $a_{b, d}$ with $d \leq \nu-k m-\ln$.

The proof is also a slight generalization of the proof of Proposition (5.9) in [8].

We recall that the global moduli space of foliations is obtained from the local one by considering the weighted action of $\mathbb{C}^{*}$ on $\mathcal{A}$ which is also the flow of $X_{0,0}$.

Proposition 2.5. The family of vector fields defined by

$$
\widetilde{X_{m, n}}=a_{2, k l+1}^{k m+l n} X_{m, n},
$$

commute with $X_{0,0}$. Therefore, they induced the distribution $\mathcal{C}$ on $\mathcal{M}$.

Proof. The proposition relies on the following computation. Since the $X_{0,0}$-degree of the variable $a_{1, k l+1}$ is equal to 1 , we have

$$
\begin{aligned}
{\left[r X_{0,0}, a_{2, k l+1}^{k m+l n} X_{m, n}\right] } & =r X_{0,0}\left(a_{2, k l+1}^{k m+l n}\right) X_{m, n}+a_{2, k l+1}^{k m+l n}\left[r X_{0,0}, X_{m, n}\right] \\
& =-(k m+\ln ) a_{2, k l+1}^{k m+l n} X_{m, n}+a_{2, k l+1}^{k m+l n}\left[r X_{0,0}, X_{m, n}\right]=0 .
\end{aligned}
$$

\subsection{The dimension of the generic strata.}

The dimension $\tau$ of the generic strata of the local moduli space of curves corresponds to the codimension of the distribution $\mathcal{C}$ at a generic point of $\mathcal{M}$. Therefore it suffices to determine the minimal number of vector fields $X_{m, n}$ which generate $\mathcal{C}$ and are functionnally independent. In order to describe the independence properties of the vector fields $X_{m, n}$, we introduce a decomposition by blocks of the triangle of moduli, and some related arithmetical properties:

A block $B_{i}$ in the triangle of moduli is a union of $k l$ consecutive horizontal lines from the line of index $d_{i}=i k l+1$ - see Figure (6). We denote by 
- $n_{d}$ the "dimension" of the line of index $d$ which means the number of integer points on this line.

- $N_{i}=\sum_{d=i k l+1}^{(i+1) k l} n_{d}$ the dimension of the block $B_{i}$ which is also the number of integer points in the whole block.

- $n_{i}^{\max }=\max \left\{n_{d}, d=i k l+1, \cdots(i+1) k l\right\}$ which is the greatest dimension of a line in the block $B_{i}$.

One can easily prove, by using the equations of the edges of the triangle, the following lemma ( see also figure $(6)$ :

Lemma 2.6. 1. We have: $N_{i+1}=N_{i}+k l, \quad n_{i}^{\max }=p-i$.

2. For each line of level $d$ of the block $B_{i}, n_{d}=n_{i}^{\max }$ or $n_{i}^{\max }-1$.

3. On the first line $d_{i}$ of the block $B_{i}$, the number $n_{d_{i}}$ reach the maximum, indeed, $n_{d_{i}}$ is equal to $n_{i}^{\max }$.

Let $\nu\left(X_{m, n}\right)=k m+l n+k l+1$ be the order of $X_{m, n}$. We denote by:

- $q_{d}$ the number of vector fields $X_{m, n}$ such that $\nu\left(X_{m, n}\right)=d$

- $Q_{i}=\sum_{d=i k l+1}^{(i+1) k l} q_{d}$

- $q_{i}^{\max }=\max \left\{q_{d}, d=i k l+1, \cdots(i+1) k l\right\}$.

One can check a similar result to $(2.6)$ :

Lemma 2.7. 1. We have: $Q_{i+1}=Q_{i}+k l, q_{i}^{\max }=i$.

2. For each line of level $d$ of the block $B_{i}, q_{d}=q_{i}^{\max }$ or $q_{i}^{\max }-1$.

3. On the first line $d_{i}$ of the block $B_{i}$, the number $q_{d_{i}}$ reach the maximum $q_{i}^{\max }$.

We consider the maximal sequence of blocks $B_{i}$ such that $q_{i}^{\max }=i<n_{i}^{\max }=p-i$, i.e. the sequence $B_{1}, \cdots B_{] p / 2]}$, where $] p / 2$ ] is the strict integer part of $p / 2$. We called critical block, the block $B \frac{p}{2}$ when $p$ is even or the unique block that appears $B_{1}$ when $p=1$. This block is going to be analyzed independently.

Lemma 2.8. Suppose that $p \geq 2$. Then the $Q_{1}$ vector fields $X_{m, n}$ such that $\nu\left(X_{m, n}\right)$ belongs to a level of the block $B_{1}$ are independent.

Proof. Since $p \geq 2$ then, we have $q_{1}^{\max }=1<n_{1}^{\max }=p-1$. We consider a relation $\sum \alpha_{m, n} X_{m, n}=0$ between the vector field $X_{m, n}$. By projection on the level $d_{1}$, we have $\alpha_{0,0} X_{0,0}^{\left(d_{1}\right)}=0$ and since $X_{0,0}^{\left(d_{1}\right)} \not \equiv 0, \alpha_{0,0}=0$. Since we have at most one vector field on each level of $B_{1}$, by iteration we get $\alpha_{m, n}=0$ for all the $(m, n)$ such that $\nu\left(X_{m, n}\right)$ belongs to $\left\{d_{1}, \cdots d_{2}-1\right\}$.

Using same kind of arguments, we can extend this result to the union of blocks of index less than the critical block:

Lemma 2.9. The $Q_{1}+\cdots+Q_{] p / 2]}$ vector fields $X_{m, n}$ such that $\nu\left(X_{m, n}\right)$ belongs to a level of the sequence of blocks $B_{1}, B_{2}, \ldots, B_{\rceil \frac{p}{2}}$ are functionnally independent.

Proof. We consider a relation $\sum \alpha_{m, n} X_{m, n}=0$ between these vector fields such that $\nu\left(X_{m, n}\right)$ belongs to $\left\{d_{1}, \cdots d_{] p / 2]}+k l-1\right\}$. If we project this relation on the first block we have from the previous lemma $\alpha_{m, n}=0$ for all the $(m, n)$ such that $\nu\left(X_{m, n}\right)$ belongs to $\left\{d_{1}, \cdots d_{2}-1\right\}$. By projection on the first line of $B_{2}$, we obtain

$$
\alpha_{l, 0} X_{l, 0}^{\left(d_{2}\right)}+\alpha_{0, k} X_{0, k}^{\left(d_{2}\right)}=0 .
$$


We claim that these two vector fields $X_{l, 0}^{\left(d_{2}\right)}$ and $X_{0, k}^{\left(d_{2}\right)}$ are independent. The projection of the vector field $X_{0,0}$ on the level $d_{1}$ is given by

$$
X_{0,0}^{\left(d_{1}\right)}=\sum_{b=1}^{p-1} \Gamma_{\phi\left(b, d_{1}\right)} e_{\phi\left(b, d_{1}\right)} .
$$

Now, using the action of $x$. and $y$. on the basis $\left\{e_{\phi(b, d)}\right\}$, we find

$$
\begin{aligned}
x^{l} \cdot e_{\Phi\left(b, d_{1}\right)} & =e_{\Phi\left(b+1, d_{2}\right)}, \text { for } b=1, \ldots, p-1 \\
x^{l} \cdot e_{\Phi\left(p, d_{1}\right)} & =0 . \\
y^{k} \cdot e_{\Phi\left(b, d_{1}\right)} & =e_{\Phi\left(b, d_{2}\right)}, \text { for } b=2 \cdots p \\
y^{k} \cdot e_{\Phi\left(1, d_{1}\right)} & =0 .
\end{aligned}
$$

Therefore the components of $X_{l, 0}$ on the $e_{\Phi\left(b, d_{2}\right)}$ are the first $p-2$ components of $X_{0,0}^{\left(d_{1}\right)}$ on the $e_{\Phi\left(b, d_{1}\right)}$ and the components of $X_{0, k}$ on the $e_{\Phi\left(b, d_{2}\right)}$ are the last $p-2$ components of $X_{0,0}^{\left(d_{1}\right)}$. Thus the first $2 \times 2$ determinant between these components is

$$
\Delta_{2}=\left|\begin{array}{ll}
\Gamma_{\phi\left(1, d_{1}\right)} & \Gamma_{\phi\left(2, d_{1}\right)} \\
\Gamma_{\phi\left(2, d_{1}\right)} & \Gamma_{\phi\left(3, d_{1}\right)}
\end{array}\right|
$$

This determinant cannot vanish. Indeed, according to the formula (7) obtained in the proof of Lemma (1.12), we have

$$
\frac{\partial}{\partial a_{b, d_{0}}}=\sum_{b^{\prime}} \lambda_{b} \mu_{b}^{b^{\prime}} e_{\Phi\left(b^{\prime}, d_{0}\right)}
$$

formula which is still valid for any $d$ according to Lemma (1.14). Therefore we have:

$$
\begin{aligned}
X_{0,0}^{\left(d_{1}\right)} & =\sum_{b} a_{b, d_{1}} \frac{\partial}{\partial a_{b, d_{1}}} \\
& =\sum_{b} a_{b, d_{1}}\left(\sum_{b^{\prime}} \lambda_{b}\left(\mu_{b}\right)^{b^{\prime}} e_{\Phi\left(b^{\prime}, d_{1}\right)}\right) \\
& =\sum_{b^{\prime}}\left(\sum_{b} a_{b, d_{1}} \lambda_{b}\left(\mu_{b}\right)^{b^{\prime}}\right) e_{\Phi\left(b^{\prime}, d_{1}\right.}
\end{aligned}
$$

which proves that for any $b^{\prime}$,

$$
\Gamma_{\phi\left(b^{\prime}, d_{1}\right)}=\sum_{b} a_{b, d_{1}} \lambda_{b}\left(\mu_{b}\right)^{b^{\prime}}
$$

From this expression, we conclude that the determinant $\Delta_{2}$ is a non trivial quadratic form in the variables $a_{b, d_{1}}$ (with rational coefficients in $a_{b, d_{0}}$ ), and therefore cannot vanish identically.

On the following levels $d$ of the second block, either we have only one vector field $X_{m, n}$ such that $\nu\left(X_{m, n}\right)=d$, and as previously we obtain $\alpha_{m, n}=0$, or we have two vector fields $X_{m, n}$ and $X_{m^{\prime}, n^{\prime}}$ such that $\nu\left(X_{m, n}\right)=\nu\left(X_{m^{\prime}, n^{\prime}}\right)=d$. The same argument as before allows us to conclude that $X_{m, n}^{(d)}$ and $X_{m^{\prime}, n^{\prime}}^{(d)}$ are independent: by using the action of the monomials $x^{m} y^{n}$ and $x^{m^{\prime}} y^{n^{\prime}}$, their components on the basis $\left(e_{\Phi(b, d)}\right)$ are the first $p-2$ components (resp. the last $p-2$ components) of $X_{0,0}^{\left(d_{1}\right)}$. 
On the upper blocks, we can prove the independence of the projection by considering a similar determinant as before

$$
\Delta_{r}=\left|\begin{array}{cccc}
\Gamma_{\phi\left(1, d_{1}\right)} & \Gamma_{\phi\left(2, d_{1}\right)} & \cdots & \Gamma_{\phi\left(r, d_{1}\right)} \\
\Gamma_{\phi\left(2, d_{1}\right)} & \Gamma_{\phi\left(3, d_{1}\right)} & \cdots & \Gamma_{\phi\left(r+1, d_{1}\right)} \\
\vdots & \vdots & & \vdots \\
\Gamma_{\phi\left(r, d_{1}\right)} & \Gamma_{\phi\left(r+1, d_{1}\right)} & \cdots & \Gamma_{\phi\left(2 r, d_{1}\right)}
\end{array}\right|
$$

From the formula (13) we deduce that $\Delta_{r}$ is an homogeneous expression of degree $r$ in the variables $a_{b, d_{1}}$ which cannot identically vanish.

Remark 2.10. Consider a block $B_{i}$ such that $q_{i}^{\max }>n_{i}^{\max }$. For each line of index $d$ of this block, since $q_{d}=q_{i}^{\max }$ or $q_{i}^{\max }-1$, we have: $q_{d} \geq n_{i}^{\max } \geq n_{d}$. According to the previous functional independence of the vector fields $X_{m, n}$, we can conclude that in this case their action is transitive on such a block and the following ones.

Consider finally the critical block $B_{i}$ such that $q_{i}^{\max }=i=n_{i}^{\max }=p-i$, i.e. the block of index $i$ such that $2 i=p$ or $B_{1}$ when $p=1$. In figure (6), this block in the second one but in figure (1), since $p=1$, this block is the sole block $B_{1}$.

In this case, the integers $n_{d}-q_{d}$ for $d=d_{i}, \cdots d_{i}+k l-1$ can only take the values +1 , 0 or -1 , starting from the value 0 . Therefore on this first level the action of the $X_{m, n}$ is transitive. We consider the first line of this block on which $n_{d}-q_{d} \neq 0$ :

- If we have $n_{d}-q_{d}=+1$, there remains one dimension which can't be reach by the action of the $X_{m, n}$. We have to count it in the codimension of the generic leaves of $\mathcal{C}$.

- If $n_{d}-q_{d}=-1$, the action of the vector fields $X_{m, n}$ is transitive on this level. Furthermore we have an extra vector field $X_{m, n}$ such that $\nu\left(X_{m, n}\right)=d$ whose higher components will act on the higher levels. Suppose that there exists a level $d^{\prime}>d$ such that $n_{d^{\prime}}-q_{d^{\prime}}=+1$.

Lemma 2.11. The $d^{\prime}$-component of this extra vector field acts on this level independently of the other vector fields $X_{m, n}$ such that $\nu\left(X_{m, n}\right)=d^{\prime}$.

Since the proof of this lemma deserves to be developped besides the fact that it is not very relevant and quite technical, we refer to the appendix B for more details.

Therefore, in order to number the generic codimension of the distribution $\mathcal{C}$ on the critical block, we have to introduce the following non commutative sum :

Definition 2.12. Let $r_{d}$ be a sequence taking its values in $\{-1,0,+1\}$. The notation $\widetilde{\Sigma}_{d} r_{d}$ denote the value obtained by the following operations:

1. delete the values 0 ;

2. delete recursively the consecutive values $(-1,+1)$ (but not the consecutive values $(+1,-1))$;

3. after the two first steps, remains a sequence of $n$ consecutive terms with value +1 , followed by $m$ consecutive terms with value -1 . We set: $\widetilde{\sum_{d}} r_{d}=n$.

Example. In the critical block of Figure (6), the sequence of values $n_{d}-q_{d}$ is:

$$
\{0,+1,+1,0,+1,0,-1,+1,0,-1,0,-1,-1,0,-1\} \text {. }
$$


The extra vector field appearing on the $7^{\text {th }}$ position acts on the next level. The next extra vector fields are unuseful. Therefore, the number of free dimensions under the action of these vector fields is

$$
\widetilde{\sum}\{0,+1,+1,0,+1,0,-1,+1,0,-1,0,-1,-1,0,-1\}=3 .
$$

From Lemmas (2.8), (2.9), (2.11) and Remark (2.10), we obtain:

Theorem 2.13. The dimension of the generic strata of the moduli space for curves is

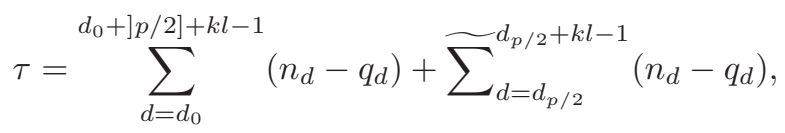

where $\left.\left.n_{d}=\right] \frac{v-k}{k}\left(\nu_{c}-d+k l\right)\right]-\left[\frac{u-l}{l}\left(\nu_{c}-d+k l\right)\left[, q_{d}\right.\right.$ is the number of positive integer solutions $(m, n)$ of the equation $k m+l n+k l+1=d$, and the second sum $\widetilde{\sum}$ is defined above and only appears if $p$ is even or if $p=1$.

Example. In the topological class $(k, l)=(3,5)$ and $p=4$ of figure $(6)$, we obtain $\tau=35$.

\subsection{Normal forms for curves.}

Theorem 2.14. We consider the reduced normal form

$$
N_{a}=x y \prod_{b=1}^{p}\left(y^{k}+\sum_{\Phi(b, d) \in \mathbb{T}} a_{b, d} m^{d}\right)
$$

obtained for the classification of foliations defined by topologically quasi-homogeneous functions. We obtain a generic unique normal form $N_{b}, b \in \mathbb{C}^{\tau}$ for the classification of curves by performing the following operations on $N_{a}$ :

1. we set: $a_{1, k l+1}=1$;

2. for each level $d$ in a block $\left.\left.B_{i}, i \leq\right] p / 2\right]$, we set $a_{b, d}=0$ for the first $q_{d}$ coefficients starting from the rightside of the line $d$;

3. for each level in the critical block $B_{p / 2}$ (which appears if $p$ is even), we consider the sequence of number $n_{d}-q_{d}$ (recall that in this block we have $n_{d} \in\{1,2\}, q_{d} \in\{1,2\}$, and $n_{d}-q_{d} \in\{-1,0,+1\}$.

- if $n_{d}-q_{d}=0$, we vanish all the coefficients of the line;

- if $n_{d}-q_{d}=+1$, we set $a_{b, d}=0$ for the first coefficient starting from the rightside of the line $d$;

- for the first lines such that $n_{d}-q_{d}=-1$ and encountered in the sequence on some line $d$, we set $a_{b, d}=0$ for the unique coefficient on this line. Furthermore, we set $a_{b, d^{\prime}}=0$ for the second coefficient on the next line $d^{\prime}>d$ such that $n_{d^{\prime}}-q_{d^{\prime}}=+1$, if such line exists.

- for the last line such that $n_{d}-q_{d}=-1$ without upper line $d^{\prime}$ such that $n_{d^{\prime}}-q_{d^{\prime}}=$ +1 we set $a_{b, d}=0$ for the unique coefficient on this line.

4. for each level $d$ in a block $\left.\left.B_{i}, i>\right] p / 2\right]$, and every index $b$, we set $a_{b, d}=0$.

Proof. Since the projection $X_{0,0}^{\left(d_{1}\right)}$ of $X_{0,0}$ on the first line of the block $B_{1}$ is the radial vector field in the variables $a_{b, d_{1}}$, its flow acts by homothety on this level and we can make use of its action to normalize one coefficient to the value 1 . We choose the first one starting from the right side. 
On all the higher levels of index $d>d_{1}$ and for the $q_{d}$ vector fields $X_{m, n}$ such that $\nu\left(X_{m, n}\right)=d$, we have

$$
X_{m, n}^{(d)}=\sum_{b} \Gamma_{m, n}\left(a_{d_{0}}, a_{d_{1}}\right) \frac{\partial}{\partial a_{b, d}}
$$

in which $\Gamma_{m, n}\left(a_{d_{0}}, a_{d_{1}}\right)$ only depends on the variables $a_{b, d_{0}}$ and $a_{b, d_{1}}$. This is a consequence of $X_{m, n}=x^{m} y^{n} \cdot X_{0,0}$ and Lemma (1.13). Therefore this vector field is constant with respect to the variables of the level $d>d_{1}$. Its flow acts by translation and we make use of this flow (and the indepence property) to vanish $q_{d}$ coefficients.

In the critical block, if there is an extra vector field $X_{m, n}$ on a line $d$ such that $n_{d}-q_{d}=$ -1 , we make use of the component $X_{m, n}^{\left(d^{\prime}\right)}$ to act on the next level $d^{\prime}$ such that $n_{d^{\prime}}-q_{d^{\prime}}=+1$. Suppose that this level is the next one $\left(d^{\prime}=d+1\right)$. This means that we have to consider the action of the second non vanishing component of $X_{m, n}$. According to Lemma (1.13), this one will depend on the variables $a_{b, d_{0}}, a_{b, d_{1}}$ and $a_{b, d_{1}+1}$. If we have to skip two lines it will depend on the variables $a_{b, d_{0}}, a_{b, d_{1}}, a_{b, d_{1}+1}$ and $a_{b, d_{1}+2}$, and so one. Therefore, it turns out that the components of $X_{m, n}^{\left(d^{\prime}\right)}$ will only depend on variables $a_{b, d}$ with $d<d^{\prime}$. Its flow still acts by translation and we make use of it to vanish the second coefficient of this line.

We give in Appendix $\mathrm{C}$ the generic normal form obtained in the topological class $(k, l)=(3,5)$ and $p=4$

\subsection{An example: the case $y^{n}+x^{n+1}$}

In, O. Zariski compute the dimension of the generic stratum of the moduli space of the curve

$$
y^{n}+x^{n+1}
$$

for $n \geq 2$. We are going to apply our strategy to recover this dimension.

Let us consider $k=n$ and $l=n+1$. In this situation, the fundamental Bézout relation is written

$$
n \cdot n-(n-1) \cdot(n+1)=1 \text {. }
$$

Thus, the triangle $\mathbb{T}$ is delimited by the two lines

$$
\begin{aligned}
j-n i & =n^{2}-n-1 \\
j-(n+1) i & =n^{2}-n-1
\end{aligned}
$$

Since there is only one branch, there is only one block and it is a critical block. From the construction of the triangle, it can be seen that the value of $n_{d}-q_{d}$ for $d_{0} \leq d \leq$ $d_{0}+n^{2}+n-1$ is

$$
\left\{\begin{array}{cl}
1 & \text { if }(\alpha-1) n+\alpha+1 \leq d \leq \alpha n-2 \\
-1 & \text { if } \alpha n+1 \leq d \leq \alpha n+\alpha+1 \\
0 & \text { else }
\end{array}\right.
$$

where $\alpha$ is an integer that goes from 1 to $n-1$. Thus, the sequence $n_{d}-q_{d}$ is the following

$$
0, \underbrace{1,1, \cdots, 1,1}_{j=1 \cdots n-2}, 0,0,-1,-1, \underbrace{1,1, \cdots, 1,1}_{j=n+3 \cdots 2 n-2}, 0,0,0,-1,-1,-1, \cdots
$$




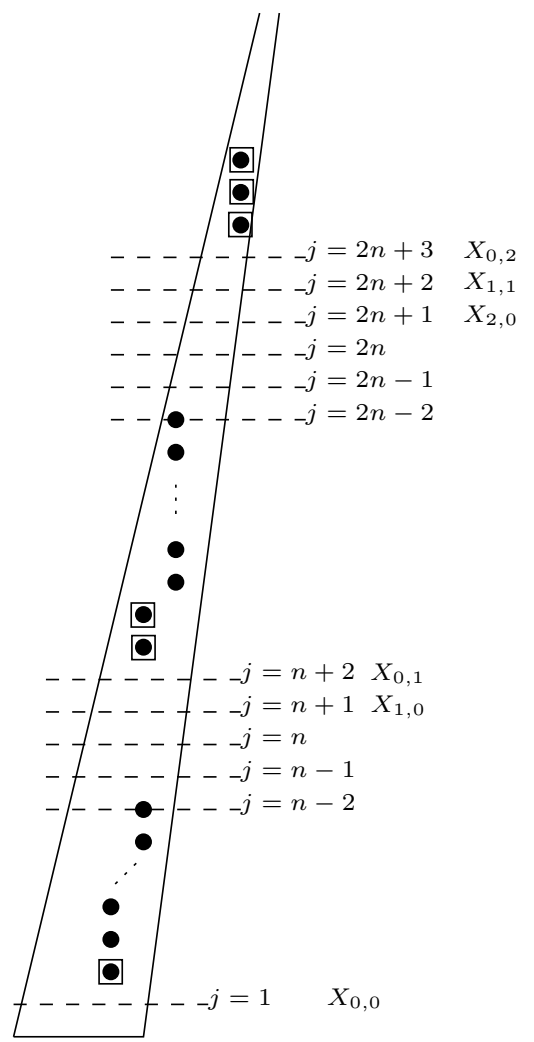

Figure 1: The case $y^{n}+x^{n+1}$

$$
\begin{aligned}
\tau & =\widetilde{\sum_{n-1}\{0,1,1, \cdots, 1,1,0,0,-1,-1, \cdots\}} \\
& =\sum_{\alpha=1}^{n-1}(n-2 \alpha-2)^{+} \\
& =\frac{(n-4)(n-2)}{4} \text { if } n \text { is even } \\
& =\frac{(n-3)^{2}}{4} \text { if } n \text { is odd }
\end{aligned}
$$

which are the formulas given in [16].

\section{Appendix}

\subsection{A: reduction of singularities of a quasi-homogeneous function}

Let $f$ be a quasihomogeneous function of weight $(k, l)$ with $p$ cuspidal branches, and multiplicities $\left(n_{0}, n_{\infty}, n_{1}, \cdots n_{p}\right)$, and $\mathcal{F}$ the foliation defined by $f$. Let $\theta_{f}$ be a vector field with isolated singularity, which defines the foliation $\mathcal{F}$. We choose the following one: let $f^{r e d}$ be the reduced function related to $f$. The one-form $f^{r e d} d f / f$ has an isolated singularity and define the same foliation as $d f$. Let $\theta_{f}$ be the dual vector field of this 
one-form for the volume form: $d x \wedge d y\left(\theta_{f}, \cdot\right)=f^{r e d} d f / f$. The components of $\theta_{f}$ are:

$$
\left(\frac{-\partial f}{\partial y}, \frac{\partial f}{\partial x}\right) / \text { g.c.d. }\left(\frac{-\partial f}{\partial y}, \frac{\partial f}{\partial x}\right) .
$$

\subsubsection{The algorithm of desingularization}

The blowing-up $E_{1}$ of $\left(\mathbb{C}^{2}, 0\right)$ endowed with the chart $(x, y)$ is the manifold defined by two charts $\left(x_{1}, y_{1}\right)$ and $\left(\overline{x_{1}}, \overline{y_{1}}\right)$ such that

$$
(x, y)=\left(x_{1}, x_{1} y_{1}\right)=\left(\overline{x_{1}} \overline{y_{1}}, \overline{y_{1}}\right) .
$$

We have $E^{-1}(0)=\left(x_{1}=0\right)=\left(\overline{y_{1}}=0\right)$. The change of charts is given by

$$
x_{1}=\overline{x_{1}} \overline{y_{1}}, y_{1}={\overline{x_{1}}}^{-1} \text {. }
$$

We will always keep these conventions:

The first coordinate of the first chart, and the second coordinate of the second chart are equations of the divisor. The center of the first chart is denoted by 0 and the center of the second one is denoted by $\infty$. In the following symbolic representation of a blowing up, we always put the first chart on the right side of the component:

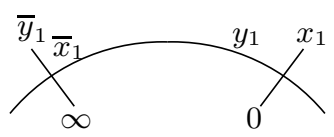

Figure 2: The atlas of one blowing up.

We denote by $E: M \rightarrow\left(\mathbb{C}^{2}, 0\right)$ the desingularization map of $f$ obtained by composition of the blowing up's. Let $D=E^{-1}(0)$ be the exceptional divisor. The reduction of singularities of the topological model

$$
f=x^{n_{0}} y^{n_{\infty}} \prod_{b=1}^{p}\left(y^{k}+a_{b} x^{l}\right)^{n_{b}}, \quad k<l, n_{0} \geq 0, n_{\infty} \geq 0, n_{b}>0
$$

is completely determined by the Euclide algorithm of the pair $(k, l)$. Indeed, a blowing-up replace any cusp of weights $(k, l)$ by a new cusp with the weights $(k, l-k)$. Therefore we perform a sequence of $q$ blowing up's until the cuspidal branches switch at infinity $(q=[l / k[+1)$. Then we perform a new sequence until the next switch and so on until getting the pair $(1,1)$. This algorithm has the following properties:

- we only have to use blowing up's of 0 or $\infty$. Therefore, the tree of the exceptional divisor is a totally ordered sequence of components, and the map $E$ is monomial in each chart.

- all the cuspidal branches follow the same infitesimal point, before the last blowing up. After this one, they appear on the same component of $D$ : we call it the principal component.

- if $n_{0} \neq 0$ or $n_{\infty} \neq 0$, the corresponding strict branches appear on the end components.

\subsubsection{Atlas of charts induced by the algorithm.}

In our present case, since $l-k \geq 0$, we have to perform a second blowing up of the origin of $\left(x_{1}, y_{1}\right)$ setting (with our convention)

$$
\left(x_{1}, y_{1}\right)=\left(x_{2}, x_{2} y_{2}\right)=\left(\overline{x_{2}} \overline{y_{2}}, \overline{y_{2}}\right) .
$$




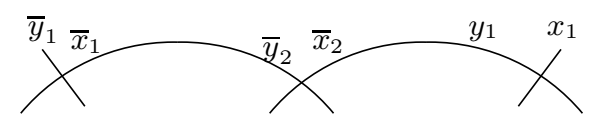

Figure 3: The atlas of two blowing up's at the origins.

We can cover the divisor by the 3 charts: $\left(\overline{x_{1}}, \overline{y_{1}}\right),\left(\overline{x_{2}}, \overline{y_{2}}\right)$ and $\left(x_{2}, y_{2}\right)$ :

At the next step, either we have to blow up the origin of the last component (we substitute $\left(x_{2}, y_{2}\right)$ by two new charts) or we have to blow up the $\infty$ point of this component (we substitute $\left(\overline{x_{2}}, \overline{y_{2}}\right)$ by two new charts). At the end of the process, we obtain $N$ components covered by $N+1$ charts. Since our divisor is totally ordered it is more convenient to replace the historical numbering of the components and the corresponding atlas with a geometrical renumbering from the right to the left side. We obtain $N+1$ charts $\left(x_{i}, y_{i}\right), i=0, \cdots N$, such that each component $D_{i}, i=1 \cdots N$ is covered by the domains $V_{i-1}$ and $V_{i}$ of the charts $\left(x_{i-1}, y_{i-1}\right)$ and $\left(x_{i}, y_{i}\right)$ around $\left(D_{i}, 0\right)$ and $\left(D_{i}, \infty\right)$. The change of charts is given by:

$$
x_{i-1}=y_{i}^{-1}, \quad y_{i-1}=x_{i} y_{i}^{e_{i}}, \quad e_{i} \in \mathbb{Z}^{\geq 1}
$$

where $-e_{i}$ is the self intersection of the component $D_{i}$. In the previous example of figure 3 , the renumbering is:

$$
\begin{aligned}
& \left(x_{4}, y_{4}\right):=\left(\overline{x_{1}}, \overline{y_{1}}\right), \quad\left(x_{3}, y_{3}\right):=\left(\overline{x_{3}}, \overline{y_{3}}\right), \quad\left(x_{2}, y_{2}\right):=\left(\overline{x_{4}}, \overline{y_{4}}\right), \\
& \left(x_{1}, y_{1}\right):=\left(x_{4}, y_{4}\right), \quad\left(x_{0}, y_{0}\right):=\left(x_{2}, y_{2}\right) .
\end{aligned}
$$

The self intersections $-e_{i}$ are: $-3,-1,-2,-3$.

Notice the $y_{N}$-axis in $\left(x_{N}, y_{N}\right)$ is the strict transform of the $y$-axis and the $x_{0}$-axis in $\left(x_{0}, y_{0}\right)$ is the strict transform of the $x$-axis.

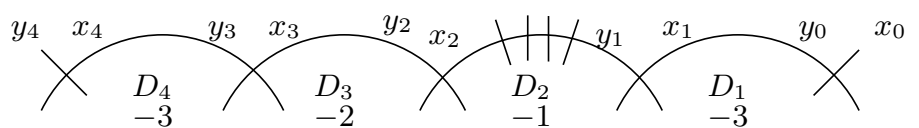

Figure 4: The atlas of the divisor for $(k, l)=(3,5)$ after renumbering.

Let $c$ be the index in $\{1, \cdots N\}$ corresponding to the principal component in the geometric numbering (in the previous example: $c=2$ ).

\subsubsection{Properties of the principal component.}

We will essentially compute in the chart $\left(x_{c}, y_{c}\right)$ centered at $\left(D_{c}, \infty\right)$. Therefore we give here the expression of $E$ in this chart and the multiplicity on $D_{c}$ :

Proposition 3.1. 1. The desingularization map $E$ is given in the chart $\left(x_{c}, y_{c}\right)$ by

$$
(x, y)=\left(x_{c}^{k-v} y_{c}^{k}, x_{c}^{l-u} y_{c}^{l}\right) .
$$

Therefore, the pull back by $E$ is given by the meromorphic expressions

$$
\left(x_{c}, y_{c}\right)=\left(\frac{x^{l}}{y^{k}}, \frac{y^{k-v}}{x^{l-u}}\right) .
$$


2. The multiplicity of $E^{*} \theta_{f}$ on the principal component $D_{c}$ of $D$ is

$$
\nu_{c}=k l p-k-l+k \varepsilon_{\infty}+l \varepsilon_{0}
$$

where $\varepsilon_{0}$ and $\varepsilon_{\infty}(=0$ or 1$)$ are the multiplicities on the axes of the reduced function $f^{r e d}$.

Proof. We prove both results by a common induction on the number of blowing up's of the minimal desingularization of $f$. For one blowing up, we have: $k=l=1, u=1, v=0$, and we have by direct computation: $\nu_{c}=p-2+\varepsilon_{0}+\varepsilon_{\infty}$. Therefore, both formulas are valid in this case.

Let $\theta_{f}$ a vector field with isolated singularity in a topological class of weights $(k, l)$, and $\nu_{c}(k, l)$ its multiplicity on the principal component. After one blowing up, the germ of foliation at the origin of the exceptional divisor is in the class $(k, l-k)$. Notice that if $u k-v l=1$ is the Bézout identity of $(k, l)$, the corresponding Bézout identity for the new pair is $(u-v) k-v(l-k)=1$. Let us suppose that the formulas of Proposition (3.1) are valid for the pair $(k, l-k)$. Therefore, after one blowing-up we have in the first chart

$$
x_{1}=x_{c}^{k-v} y_{c}^{k}, \quad y_{1}=x_{c}^{l-k-u+v} y_{c}^{l-k} .
$$

We obtain:

$$
x=x_{1}=x_{c}^{k-v} y_{c}^{k}, \quad y=x_{1} y_{1}=x_{c}^{l-u}, y_{c}^{l} .
$$

Now, we claim that

$$
\nu_{c}(k, l)=\nu_{c}(k, l-k)+p k^{2}-k+k \varepsilon_{0}
$$

from which we easily deduce the second formula. To prove this relation, let $E=E^{\prime} \circ E_{1}$ where $E_{1}$ is the first blowing up. We have:

$$
\frac{E^{*} \theta_{f}}{y_{c}^{\nu_{c}(k, l)}}=E^{\prime *}\left(\frac{E_{1}^{*} \theta_{f}}{x_{1}^{a} y_{1}^{b}}\right) \frac{E^{\prime *}\left(x_{1}^{a} y_{1}^{b}\right)}{y_{c}^{\nu_{c}(k, l)}}
$$

where $a=\nu\left(E_{1}^{*} \theta_{f}, x_{1}\right)$ and $b=\nu\left(E_{1}^{*} \theta_{f}, y_{1}\right)$. From (15) and the previous equality, we obtain

$$
\nu_{c}(k, l)=\nu_{c}(k, l-k)+a k+b(l-k) .
$$

Now we have

$$
f^{r e d} \circ E_{1}=x_{1}^{k p+1+\varepsilon_{0}} y_{1}^{\varepsilon \infty} \prod_{b=1}^{p}\left(y_{1}^{k}-a_{b} x_{1}^{l-k}\right)
$$

and thus $\nu\left(f^{r e d} \circ E_{1}, x_{1}\right)=k p+1+\varepsilon_{0}$ and $\nu\left(f^{r e d} \circ E_{1}, y_{1}\right)=\varepsilon_{\infty}$. From $E_{1}^{*} d x \wedge d y\left(E_{1}^{*} \theta_{f}, \cdot\right)=$ $E_{1}^{*}\left(f^{r e d} d f / f\right)$ we deduce:

$$
a=k p+\varepsilon_{0}-1, \quad b=0
$$

which proves the relation (16).

\subsubsection{Computing multiplicities}

We first recall the classical result which allows us to compute multiplicities of a function along $D[4]$ :

We choose the geometric numbering of the components of the exceptional divisor, and we consider the matrix of intersections $J$ defined for $i \neq j$ by $J_{i, j}=1$ if the two components $D_{i}$ and $D_{j}$ meet together, $J_{i, j}=0$ otherwise, and $J_{i, i}=-e_{i}$, where $-e_{i}$ is the self intersection of each component. Let $b_{i}$ be the number of strict branches of $f \circ E$ meeting $D_{i}$, counted with their multiplicities, and $B$ the column matrix induced by these numbers. 
Proposition 3.2. The multiplicities $m_{i}$ of $(f \circ E)$ along each $D_{i}$ define a column matrix $M$ which satisfy

$$
J M+B=0 .
$$

In the quasi-homogeneous case, since $D=D_{1} \cup \cdots \cup D_{c-1} \cup D_{c} \cup D_{c+1} \cup \cdots \cup D_{N}$, the column matrix $B$ is here: $\left(n_{0}, 0, \cdots, 0, n, 0, \cdots 0, n_{\infty}\right)^{t}$, where $n=\sum_{b=1}^{p} n_{b}$ is on index $c$. The intersection matrix is given by:

$$
J=\left(\begin{array}{cccccccc}
-e_{1} & 1 & 0 & \ldots & & & & 0 \\
1 & -e_{2} & 1 & 0 & \ldots & & & 0 \\
0 & 1 & -e_{3} & 1 & 0 & \ldots & & 0 \\
\vdots & & & & & & & \\
0 & \cdots & & & 0 & 1 & -e_{N-1} & 1 \\
0 & \cdots & & & & 0 & 1 & -e_{N}
\end{array}\right)
$$

Therefore we obtain the multiplicities of $f$ by $M=-J^{-1} B$ (see example below). We will also need to compute the multiplicities of the desingularized foliation, i.e. of the vector field $E^{*} \theta_{f}$, where $\theta_{f}$ is the vector field (14) with isolated singularity, defining the foliation $\mathcal{F}$.

Proposition 3.3. The multiplicities $\nu_{i}$ of $E^{*} \theta_{f}$ along each component $D_{i}$ define a column matrix $N$ which satisfy

$$
J N+C=0,
$$

where $C=\left(\varepsilon_{0}-1,0, \cdots, 0, p, 0, \cdots 0, \varepsilon_{\infty}-1\right)^{t}$, with $p$ on index $c, \varepsilon_{0}=1$ if $n_{0} \neq 0, \varepsilon_{0}=0$ otherwise, $\varepsilon_{\infty}=1$ if $n_{\infty} \neq 0, \varepsilon_{\infty}=0$ otherwise.

Proof. Let $V=\left(v_{i}\right)$ be the multiplicities of $E^{*} d x \wedge d y$ along each $D_{i}$. From

$$
E^{*}(d x \wedge d y)\left(E^{*} \theta, \cdot\right)=\left(f^{r e d} \circ E\right) d(f \circ E) /(f \circ E)
$$

we obtain:

$$
v_{i}+\nu_{i}=r_{i}+\left(m_{i}-1\right)-m_{i}=r_{i}-1
$$

where $r_{i}=\nu\left(f^{r e d} \circ E, D_{i}\right)$. We consider the "axis function": $a=x y$. Let $A=\left(a_{i}\right)$ be the column matrix of multiplicities of $a \circ E$ along each $D_{i}$. We claim that $v_{i}=a_{i}-1$. Indeed, let $\left(x_{i}, y_{i}\right)$ be the chart induced by $(x, y)$ and $E$ around the origin of $D_{i}$. Since $E$ is here monomial in these coordinates, there exist positive integers $p, q, r, s$, such that:

$$
E^{*} d x \wedge d y=a \circ E \cdot E^{*}\left(\frac{d x}{x} \wedge \frac{d y}{y}\right)=a \circ E \cdot(p s-q r) \frac{d x_{i}}{x_{i}} \wedge \frac{d y_{i}}{y_{i}}
$$

from which we deduce $v_{i}=a_{i}-1$. Therefore we obtain $A+N=R$, where $R$ is the matrix of multiplities of $\left(f^{r e d} \circ E\right)$. Now, from the previous proposition we have: $J R=B^{\text {red }}$, with $B^{r e d}=\left(\varepsilon_{0}, 0, \cdots, 0, p, 0, \cdots 0, \varepsilon_{\infty}\right)^{t}$ and $J A=B^{\prime}$ where $B^{\prime}$ is the column such that $b_{i}^{\prime}=1$ for $i=1$ or $i=N$ and $b_{i}^{\prime}=0$ otherwise. We obtain:

$$
J N=J(R-A)=B^{r e d}-B^{\prime}=C .
$$

Example. For $(k, l)=(3,5)$, the matrix of intersections is:

$$
J=\left(\begin{array}{cccc}
-3 & 1 & 0 & 0 \\
1 & -2 & 1 & 0 \\
0 & 1 & -1 & 1 \\
0 & 0 & 1 & -3
\end{array}\right)
$$


and we have $B=\left(n_{0}, 0, n, n_{\infty}\right)^{t}$, where $n=\sum_{b=1}^{p} n_{b}$, and $C=\left(\varepsilon_{0}-1, p, 0, \varepsilon_{\infty}-1\right)$. Therefore we obtain:

$$
M=\left(\begin{array}{c}
2 n_{0}+n_{\infty}+5 n \\
5 n_{0}+3 n_{\infty}+15 n \\
3 n_{0}+2 n_{\infty}+9 n \\
n_{0}+n_{\infty}+3 n
\end{array}\right) ; \quad N=\left(\begin{array}{c}
2 \varepsilon_{0}+\varepsilon_{\infty}+5 p-3 \\
5 \varepsilon_{0}+3 \varepsilon_{\infty}+15 p-8 \\
3 \varepsilon_{0}+2 \varepsilon_{\infty}+9 p-5 \\
\varepsilon_{0}+\varepsilon_{\infty}+3 p-2
\end{array}\right)
$$

The multiplicity of the foliation on the principal component $D_{2}$ is

$$
\nu_{c}=5 \varepsilon_{0}+3 \varepsilon_{\infty}+15 p-8
$$

\subsubsection{Properties of the sheaf $\Theta_{\mathcal{F}}$.}

A chain of $D$ is a connected sequence of components $D_{i}$ which meet exactly two others components. In the quasi-homogeneous case, $D$ contains two chains: $\left\{D_{2}, \cdots D_{c-1}\right\}-$ the right hand chain- and $\left\{D_{c+1}, \cdots D_{N-1}\right\}$-the left hand chain-.

Let $W_{0}$ be the union of the charts domains $V_{1}, V_{2}, \ldots V_{c-1}: W_{0}$ covers $D_{1} \cup \cdots \cup D_{c}$ excepted a small disc around 0 in $D_{1}$ and a small disc around $\infty$ in $D_{c}$. In a similar way, we consider $W_{\infty}=V_{c} \cup \cdots \cup V_{N-1}$, covering $D_{c} \cup \cdots \cup D_{N}$ excepted small discs around 0 in $D_{c}$ and around $\infty$ in $D_{N}$.

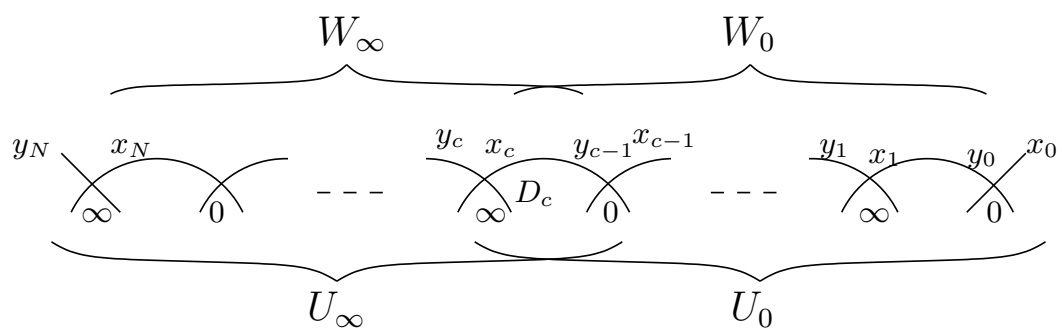

Figure 5: Open sets $W_{\infty}, W_{0}, U_{\infty}$ and $U_{0}$.

Proposition 3.4. There exists a global section $T_{0}$ (resp. $T_{\infty}$ ) of the sheaf $\Theta_{\mathcal{F}}$ of germs of vector fields tangent to $E^{*} \mathcal{F}$ on $W_{0}$ (resp. $W_{\infty}$ ) which admits only isolated singularities.

Proof. From the previous relation $J N+C=0$, we obtain :

$$
\nu_{i-1}-e_{i} \nu_{i}+\nu_{i+1}=0, \quad i=2, \cdots c-1 .
$$

We claim that the vector fields with isolated singularities

$$
\theta_{i}=\frac{E^{*} \theta_{f}}{x_{i}^{\nu_{i}} y_{i}^{\nu_{i+1}}}, i=1, \cdots c-1
$$

glue together defining a global section $T_{0}$ of $\Theta_{\mathcal{F}}$ on $W_{0}$. Indeed, using the change of two consecutive charts, we have

$$
x_{i}^{\nu_{i}} y_{i}^{\nu_{i+1}}=x_{i-1}^{\nu_{i} e_{i}} y_{i-1}^{\nu_{i}} x_{i-1}^{-\nu_{i+1}}=x_{i-1}^{\nu_{i-1}} y_{i-1}^{\nu_{i}}
$$

which proves that $\theta_{1}=\theta_{2}=\cdots=\theta_{c-1}$ on their common domains.

Corollary 3.5. We have $H^{1}\left(W_{0}, \Theta_{\mathcal{F}}\right)=H^{1}\left(W_{\infty}, \Theta_{\mathcal{F}}\right)=0$. 
Proof. The previous section $T_{0}$ with isolated singularities allows us to identify the sheaf $\left.\Theta_{\mathcal{F}}\right|_{W_{0}}$ to $\left.\mathcal{O}_{M}\right|_{W_{0}}$. Since the Chern class of each branch is negative, one can easily check that $H^{1}\left(W_{0}, \mathcal{O}_{M}\right)=0$. The proof is similar for $W_{\infty}$.

Proposition 3.6. Let $U_{0}$ be an open set covering $D_{1}, \cdots, D_{c}$ excepted a small disc in $D_{c}$ around $\left.D_{c} \cap D_{c+1}\right)$, and $U_{\infty}$ a similar domain for the left hand side. We have

$$
H^{1}\left(U_{0}, \Theta_{\mathcal{F}}\right)=H^{1}\left(U_{\infty}, \Theta_{\mathcal{F}}\right)=0 .
$$

Proof. On can suppose that $U_{0}=V_{0} \cup W_{0}$ where $V_{0}$ is the domain of $\left(x_{0}, y_{0}\right)$ at $\left(D_{1}, 0\right)$. From corollary $(3.5)$, we have $H^{1}\left(W_{0}, \Theta_{\mathcal{F}}\right)=0$. We also have $H^{1}\left(V_{0}, \Theta_{\mathcal{F}}\right)=0$ since we can choose a vector field with isolated singularities on $V_{0}$ (or without singularities if there is no invariant branch in $\left.V_{0}\right)$. Therefore we obtain:

$$
H^{1}\left(U_{0}, \Theta_{\mathcal{F}}\right)=\frac{\Theta_{\mathcal{F}}\left(V_{0} \cap W_{0}\right)}{\Theta_{\mathcal{F}}\left(V_{0}\right) \oplus \Theta_{\mathcal{F}}\left(W_{0}\right)} .
$$

Let $T_{0}$ be the vector field on $V$ given by (3.4). Let $\left(x_{0}, y_{0}\right)$ and $\left(x_{1}, y_{1}\right)$ be the charts induced by $(E,(x, y))$ on $D_{1}$ according to the previous notations and $\left(x_{0}, y_{0}\right)=\varphi\left(x_{1}, y_{1}\right)=$ $\left(x_{1}^{e_{1}} y_{1}, x_{1}^{-1}\right)$ the change of charts. We have:

$$
\begin{aligned}
& \Theta_{\mathcal{F}}\left(V_{0} \cap W_{0}\right)=\left\{g\left(x_{1}, y_{1}\right) \cdot T_{0}, g \text { holomorphic in } x_{1}\right\} \\
& \Theta_{\mathcal{F}}\left(W_{0}\right)=\left\{g\left(x_{1}, y_{1}\right) \cdot T_{0} \in \Theta_{\mathcal{F}}\left(V_{0} \cap W_{0}\right), g \text { has an holomorphic extension in } W_{0}\right\} \\
& \Theta_{\mathcal{F}}\left(V_{0}\right)=\left\{g\left(x_{1}, y_{1}\right) \cdot T_{0} \in \Theta_{\mathcal{F}}\left(V_{0} \cap W_{0}\right), \varphi^{*}\left(g \cdot T_{0}\right) \text { holomorphic at }\left(D_{0}, 0\right)\right\}
\end{aligned}
$$

We set: $g\left(x_{1}, y_{1}\right)=\sum_{i \geq 0, j \in \mathbb{Z}} a_{i, j} x_{1}^{i} y_{1}^{j}$. The existence of an holomorphic extension of $g$ along $W_{0}$ gives us the necessary condition $j \geq 0$. On the other side we have

$$
\varphi^{*}\left(\theta_{0}\right)=\frac{E^{*} T_{0}}{y_{0}^{\nu_{0}}} x_{0}
$$

The vector field $\frac{E * T_{0}}{y_{0}^{\nu 0}}$ is non singular on a neighborhood of $\left(D_{1}, 0\right)$ if there is no invariant branch at $\left(D_{1}, 0\right)$ and vanishes on this axis with some positive multiplicity $\nu$ otherwise. Therefore the condition $\varphi^{*}\left(g \cdot T_{0}\right)$ is holomorphic at $\left(\left(D_{1}, 0\right)\right)$ gives rise to: $e_{1} i-j+1+\nu \geq 0$. The two conditions $j \geq 0$ and $j \leq e_{1} i+1+\nu$ vanish any element $(i, j) \in \mathbb{Z}^{+} \times \mathbb{Z}$ in the quotient.

\subsection{B: complement on the study of the vector fields $X_{m, n}$}

In this appendix, we study the independance properties of the family of vector fields $X_{m, n}$. We consider the following decomposition

$$
\begin{aligned}
X_{0,0} & =\sum_{d} \sum_{b}(d-k l) a_{b, d} \frac{\partial}{\partial a_{b, d}} \\
& =\sum_{i, j \geq 1} \underbrace{\left(\sum_{0 \leq d-k l \leq j} \sum_{b}(d-k l) a_{b, d} A_{i, j}^{b, d}(a)\right)}_{\Gamma_{i, j}(a)} e_{i, j} .
\end{aligned}
$$

Proposition 3.7. The functions $\Gamma_{i, j}(a)$ are algebraically independent. 
Proof. Let us decompose the coefficient $\Gamma_{i, j}(a)$

$$
\Gamma_{i, j}(a)=\underbrace{\sum_{b} j a_{b, k l+j} A_{i, j}^{b, k l+j}(a)}_{L_{i, j}(a)}+\underbrace{\sum_{0 \leq d-k l<j} \sum_{b}(d-k l) a_{b, d} A_{i, j}^{b, d}(a)}_{R_{i j}(a)} .
$$

Following, the lemma (1.13) the function $A_{i, j}^{b, k l+j}(a)$ depends only on the variables $a_{b, d^{\prime}}$ with $0 \leq d^{\prime}-k l \leq j-(k l+j-k l)=0$. Thus, it depends only on the variables $a_{b, k l}$. The expression $R_{i j}$ depends only on the variables $a_{b, d^{\prime}}$ where $d^{\prime}$ satisifies

$$
0 \leq d^{\prime}-k l \leq j-(d-k l) \Longrightarrow d^{\prime} \leq j+k l-(d-k l)<j+k l .
$$

In view of (1.12), for a fixed value of $j$, the linear forms $L_{i, j}$ are independent since their determinant is $\operatorname{det}\left(\left|A_{i, j}^{b, k l+j}(a)\right|_{i, b}\right) \neq 0$. Thus, they are also algebraically independent. Now, let us consider a polynomial function $P\left(\left\{X_{i, j}\right\}_{(i, j) \in \mathbb{T}}\right)$ where the $X_{i, j}^{\prime}$ s are some independent variables. Suppose that $P$ induces an algebraic relation between the functions $\Gamma_{i, j}(a)$

$$
P\left(\Gamma_{i, j}(a)\right)=0 .
$$

Let $J$ be the greatest integer such that there exist a point $(i, J)$ in $\mathbb{T}$ and denote by $\left\{\left(i_{0}, J\right),\left(i_{1}, J\right), \cdots,\left(i_{q}, J\right)\right\}$ the family of points in $\mathbb{T}$ at the level $J$. The relation $P$ is written

$$
P\left(\left\{\Gamma_{i, j}(a)\right\}_{j<J}, L_{i_{0}, J}(a)+R_{i_{0}, J}(a), \ldots, L_{i_{q}, J}(a)+R_{i_{q}, J}(a)\right)=0 .
$$

We fix all the variables $a_{b, d}$ with $d-k l<J$ at a generic value. Then, the above relation becomes an algebraic relation between the affine forms $L_{i, J}(a)+R_{i, J}(a)$. Let us decompose the relation $P$ as follows

$$
P=\sum_{I \subset\left\{\left(i_{k}, J\right)\right\}_{k=0 . . q}} Q_{I}\left(X_{i, j}\right) X_{I}
$$

where $X_{I}=\prod_{(i, J) \in I} X_{i, J}$. Here, $Q_{I}$ depends only on the variables $X_{i, j}$ with $j<J$. Since, the affine form $L_{i, J}(a)+R_{i, J}(a)$ are algebraiclly independant, for any $I$, we have

$$
Q_{I}\left(\Gamma_{i, j}(a)\right)=0
$$

which are algebraic relations between the function $\Gamma_{i, j}(a)$ with $j<J$. Therefore, an inducing argument ensures that $P$ has to be the trivial relation, which proves the theorem.

We equipped the $\mathbb{C}$-space Vect $\left\{e_{i, j}\right\}_{(i, j) \in \mathbb{T}}$ with an hermitian product defined by

$$
\left\langle e_{i, j}, e_{i^{\prime}, j^{\prime}}\right\rangle=\delta_{(i, j),\left(i^{\prime}, j^{\prime}\right)}
$$

Corollary 3.8. Consider the subspace Vect $\left\{e_{i_{1}, j_{1}}, \ldots, e_{i_{q}, j_{q}}\right\}$ and the orthogonal projection of a sub-family $X_{m_{1}, n_{1}}, \ldots, X_{m_{q}, n_{q}}$ on this subspace. Suppose that for any $e_{i_{q}, j_{q}}$, the orthogonal projection of the family $X_{m_{1}, n_{1}}, \ldots, X_{m_{q}, n_{q}}$ on $e_{i_{q}, j_{q}}$ does not identically vanish. Then the projected sub-family is generically free.

Proof. Let us write the decomposition of $X_{0,0}$ in the basis $e_{i, j}$

$$
X_{0,0}=\sum_{(i, j) \in \mathbb{T}} \Gamma_{i, j}(a) e_{i, j}
$$


The multiplication by $x$ and $y$ define an application of $\mathcal{O}$-modules whose action on the basis $e_{i, j}$ is as follows

$$
\begin{aligned}
& x \cdot e_{i, j}=e_{k-v+i, k+j} \\
& y \cdot e_{i, j}=e_{l-u+i, l+j} .
\end{aligned}
$$

Thus we have the following decomposition

$$
\begin{aligned}
X_{m, n}=x^{m} y^{n} \cdot X_{0,0} & =\sum_{(i, j) \in \mathbb{T}} \Gamma_{i, j}(a) e_{m(k-v)+n(l-u)+i, m k+l n+j} \\
& =\sum_{(i, j) \in \mathbb{T}} \Gamma_{\tau_{m, n(i, j)}}(a) e_{i, j}
\end{aligned}
$$

where $\tau_{m, n}$ is the translation of vector $m \vec{x}+n \vec{y}$. In this expression, we set $\Gamma_{\star, \star}=0$ if $(\star, \star) \notin \mathbb{T}$. The determinant of this projected family is

$$
\left|\Gamma_{\tau_{m_{r}, n_{r}}\left(i_{s}, j_{s}\right)}(a)\right| \begin{aligned}
& r=1 \ldots q \\
& s=1 \ldots q
\end{aligned} .
$$

Since the functions involved in the above determinant are algebraically independant, this determinant does not indentically vanish if and only if the determinant

$$
\left|X_{\tau_{m_{r}, n_{r}}\left(i_{s}, j_{s}\right)}\right| \begin{array}{r}
r=1 \ldots q \\
s=1 \ldots q
\end{array}
$$

is not zero. The hypothesis of the corollary ensures that in the previous determinant there is no zero row. Moreover notice that in the matrix $\left[X_{\tau_{m_{r}, n_{r}}\left(i_{s}, j_{s}\right)}\right]_{r=1 \ldots q}$ a $s=1 \ldots q$

variable $X_{i, j}$ cannot appear twice on the same row or on the same line. Suppose now that there exists a vector $\left(\lambda_{i}\right)_{i=1 . . q}$ in the kernel of this matrix which means that for any $r$ the following relation holds

$$
\sum_{s=1 . . q} \lambda_{s} X_{\tau_{m_{r}, n_{r}}\left(i_{s}, j_{s}\right)}=0
$$

Obviously, if $\tau_{m_{r}, n_{r}}\left(i_{s}, j_{s}\right) \in \mathbb{T}$ then $\lambda_{s}=0$. Since, there is no zero row in the initial matrix, then for any $s$ there exists $r$ such that $\tau_{m_{r}, n_{r}}\left(i_{s}, j_{s}\right) \in \mathbb{T}$, thus for any $s$, the coefficient $\lambda_{s}=0$, which proves the proposition.

\subsection{C: normal forms for $(k, l)=(3,5)$ and $p=4$}

According to the figure draw below, the analytical normal form for the topological class of

$$
\left(y^{3}+x^{5}\right)^{n_{1}}\left(y^{3}+a_{2} x^{5}\right)^{n_{2}}\left(y^{3}+a_{3} x^{5}\right)^{n_{3}}\left(y^{3}+a_{4} x^{5}\right)^{n_{4}} .
$$

is given by the following family of functions with 78 parameters 


$$
\begin{aligned}
N_{a}=( & \left(y^{3}+x^{5}+a_{1,16} x^{2} y^{2}+a_{1,19} x^{3} y^{2}\right)^{n_{1}} \times \\
\left(y^{3}\right. & +a_{2,15} x^{5}+a_{2,16} x^{2} y^{2}+a_{2,17} x^{4} y+a_{2,18} x^{6}+a_{2,19} x^{3} y^{2}+a_{2,20} x^{5} y \\
& +a_{2,21} x^{7}+a_{2,22} x^{4} y^{2}+a_{2,23} x^{6} y+a_{2,24} x^{8}+a_{2,25} x^{5} y^{2}+a_{2,26} x^{7} y \\
& \left.+a_{2,28} x^{6} y^{2}+a_{2,29} x^{8} y+a_{2,31} x^{7} y^{2}+a_{2,34} x^{8} y^{2}\right)^{n_{2}} \times \\
\left(y^{3}\right. & +a_{3,15} x^{5}+a_{3,16} x^{2} y^{2}+a_{3,17} x^{4} y+a_{3,18} x^{6}+a_{3,19} x^{3} y^{2}+a_{3,20} x^{5} y \\
& +a_{3,21} x^{7}+a_{3,22} x^{4} y^{2}+a_{3,23} x^{6} y+a_{3,24} x^{8}+a_{3,25} x^{5} y^{2}+a_{3,26} x^{7} y \\
& +a_{3,27} x^{9}+a_{3,28} x^{6} y^{2}+a_{3,29} x^{8} y+a_{3,30} x^{10}+a_{3,31} x^{7} y^{2}+a_{3,32} x^{9} y \\
& +a_{3,33} x^{11}+a_{3,34} x^{8} y^{2}+a_{3,35} x^{10} y+a_{3,36} x^{12}+a_{3,37} x^{8} y^{2}+a_{3,38} x^{11} y \\
& +a_{3,39} x^{13}+a_{3,40} x^{9} y^{2}+a_{3,41} x^{12} y+a_{3,43} x^{10} y^{2}+a_{3,44} x^{13} y+a_{3,46} x^{12} y^{2} \\
& \left.+a_{3,49} x^{13} y^{2}\right)^{n_{3}} \times \\
\left(y^{3}\right. & +a_{4,15} x^{5}+a_{4,17} x^{4} y+a_{4,18} x^{6}+a_{4,20} x^{5} y+a_{4,21} x^{7}+a_{4,23} x^{6} y \\
& +a_{4,24} x^{8}+a_{4,26} x^{7} y+a_{4,27} x^{9}+a_{4,29} x^{8} y+a_{4,30} x^{10}+a_{4,32} x^{9} y \\
& +a_{4,33} x^{11}+a_{4,35} x^{10} y+a_{4,36} x^{12}+a_{4,38} x^{11} y+a_{4,39} x^{13}+a_{4,41} x^{12} y \\
& +a_{4,42} x^{14}+a_{4,44} x^{13} y+a_{4,45} x^{15}+a_{4,47} x^{14} y+a_{4,48} x^{16}+a_{4,50} x^{15} y \\
& \left.+a_{4,51} x^{17}+a_{4,53} x^{16} y+a_{4,54} x^{18}+a_{4,56} x^{17} y+a_{4,59} x^{18} y\right)^{n_{4}} .
\end{aligned}
$$

Moreover, the normal forms for the generic curve are given by the 35-parameters family

$$
\begin{aligned}
N_{a}= & \left(y^{3}+x^{5}+x^{2} y^{2}\right) \times \\
\left(y^{3}\right. & +a_{2,15} x^{5}+a_{2,16} x^{2} y^{2}+a_{2,17} x^{4} y+a_{2,18} x^{6}+a_{2,19} x^{3} y^{2}+a_{2,20} x^{5} y \\
& \left.+a_{2,23} x^{6} y\right) \times \\
\left(y^{3}\right. & +a_{3,15} x^{5}+a_{3,16} x^{2} y^{2}+a_{3,17} x^{4} y+a_{3,18} x^{6}+a_{3,19} x^{3} y^{2}+a_{3,20} x^{5} y \\
& +a_{3,21} x^{7}+a_{3,22} x^{4} y^{2}+a_{3,23} x^{6} y+a_{3,24} x^{8}+a_{3,25} x^{5} y^{2}+a_{3,26} x^{7} y \\
& \left.+a_{3,28} x^{6} y^{2}+a_{3,29} x^{8} y\right) \times \\
\left(y^{3}\right. & +a_{4,15} x^{5}+a_{4,17} x^{4} y+a_{4,18} x^{6}+a_{4,20} x^{5} y+a_{4,21} x^{7}+a_{4,23} x^{6} y \\
& +a_{4,24} x^{8}+a_{4,26} x^{7} y+a_{4,27} x^{9}+a_{4,29} x^{8} y+a_{4,30} x^{10}+a_{4,32} x^{9} y \\
& \left.+a_{4,33} x^{11}+a_{4,35} x^{10} y\right) .
\end{aligned}
$$




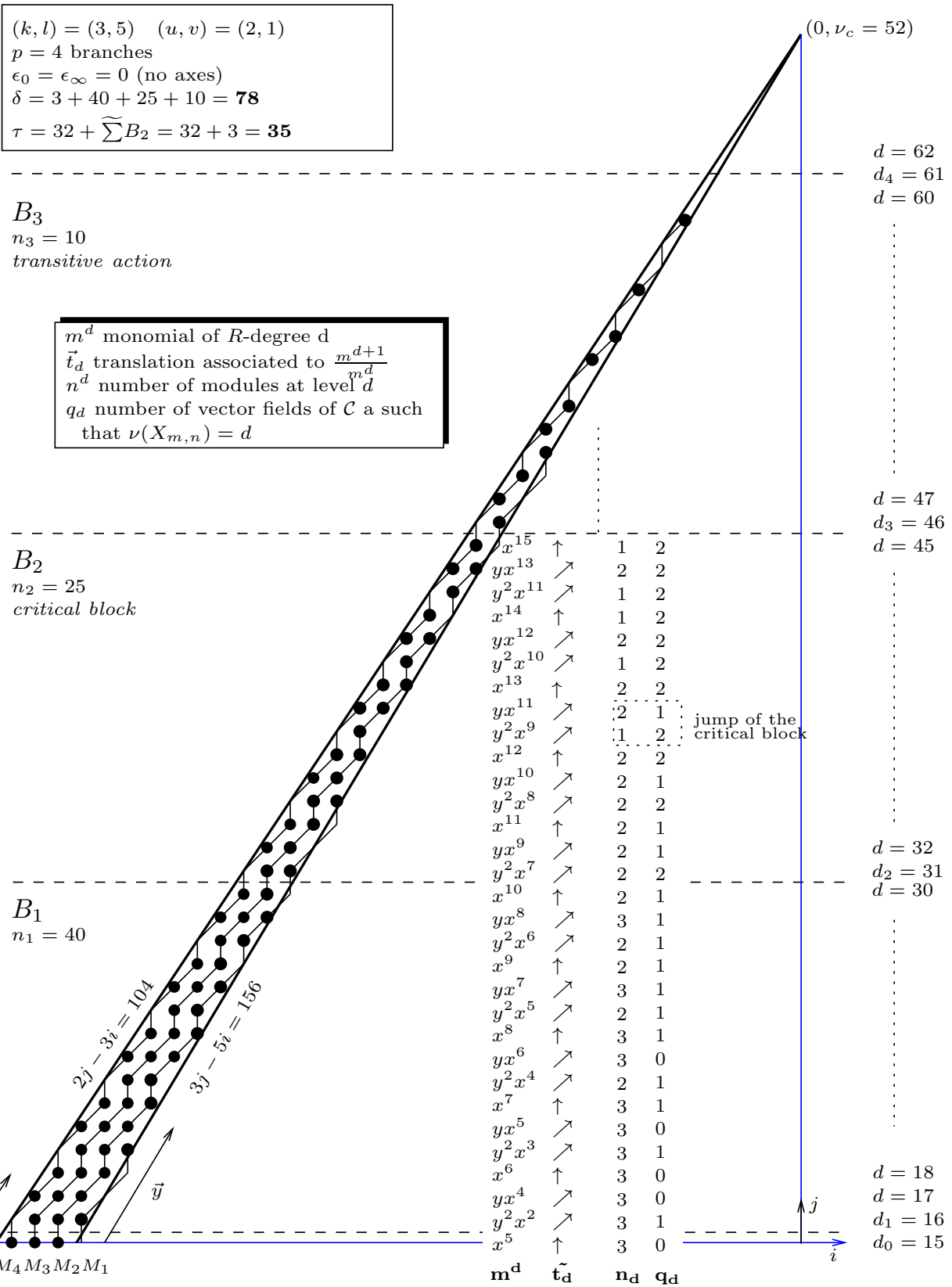

Figure 6: Moduli triangle of the topological class $(k, l)=(3,5)$ and $p=4$ 


\section{References}

[1] M. Berthier, D. Cerveau and R. Meziani, Transformations isotropes des germes de feuilletages holomorphes J. Math. Pures Appl. (9) 781999

[2] Briançon, J. and Granger, M. and Maisonobe, Ph., Le nombre de modules du germe de courbe plane $x^{a}+y^{b}=0$, Math. Ann. $27919883535-551$

[3] C. Camacho, A. Lins Neto and P. Sad, Topological invariants and equidesingularization for holomorphic vector fields J. Differential Geom. 2019841

[4] D. Eisenbud and W.D. Neumann, Three-dimensional link theory and invariants of plane curve singularities, ANN. Math. Stud. 101 (1985), Princeton University Press.

[5] Hefez, A. and Hernandes, M., Analytic classification of plane branches up to multiplicity 4, J. Symbolic Comput. 4420096

[6] Hefez, A. and Hernandes, M. , The analytic classification of plane branches, Bull. Lond. Math. Soc. 4320112 289-298.

[7] A. Hefez and Hernandes M. Standard bases for local rings of branches and their modules of differentials, J. Symbolic Comput. 42 (2007).

[8] Y. Genzmer and E. Paul - Normal forms of foliations and curves defined by a function with a generic tangent cone, Moscow math. jour. 11 (2011), 1, p. 41-72.

[9] J.M. Granger - Sur un espace de modules de germe de courbe plane, Bull. Sc. math. 103 (1979), 2é série p. 3-16.

[10] J. N. Mather and S. S. T. Yau. Classication of isolated hypersurface singularities by their moduli algebras. Invent. Math., 69(2) , 243-251, (1982)

[11] J.F. Mattei - Quasihomogénité et équiréductibilité de feuilletages holomorphes en dimension 2, Astérisque 261 S.M.F. (2000), p. 253-276.

[12] J.F. Mattei - Modules de feuilletages holomorphes singuliers: I équisingularité, Invent. Math. 103 (1991), p. 297-325.

[13] J.F. Mattei and R. Moussu - Holonomie et intégrales premières, Ann. Sci. Ecole Norm. Sup. 13 (1980), p. 469-523.

[14] Saito, K., Quasihomogene isolierte Singularitäten von Hyperflächen Invent. Math. 14 1971

[15] A. Seidenberg - Reduction of singularities of the differential equation Ady $=B d x$, Amer. J. Math. 90 (1968).

[16] O. Zariski - Le problème des modules pour les branches planes, Hermann, Paris, seconde édition (1986), course given at the Centre de Mathématiques de l'Ecole Polytechnique (1973).

\section{Y. Genzmer}

Institut de Mathématiques de Toulouse

Université Paul Sabatier

118 route de Narbonne

31062 Toulouse cedex 9, France.

yohann.genzmer@math.univ-toulouse.fr
E. Paul

Institut de Mathématiques de Toulouse

Université Paul Sabatier

118 route de Narbonne

31062 Toulouse cedex 9, France.

emmanuel.paul@math.univ-toulouse.fr 